\title{
On Locally Trivial Deformations
}

\author{
By \\ Hubert FLENNER* and Siegmund KOSAREW** \\ Contents
}

\$1. Data of Structure Preserving Maps

§2. Polycylinder Spaces and Morphism Spaces

§3. The Representability Theorem

§. Smoothness for Deformations of Holomorphic Mappings

$\S 5$. Applications

§6. Appendix : Banach Fiber Spaces over Banach Analytic Spaces

\$7. Appendix: Extensions of Holomorphic Mappings

References

\section{Introduction}

This paper originated from a question of G. M. Greuel and $U$. Karras whether any compact complex space $X$ admits a locally trivial semiuniversal deformation

$$
X \longrightarrow \mathscr{X} \longrightarrow(S, 0) \text {. }
$$

Here "locally trivial" and "locally trivial semiuniversal" means the following: $\mathscr{X}$ is called locally trivial over $S$, if for every $x \in X$ the deformation germ

$$
(\mathscr{X}, x) \longrightarrow(S, 0)
$$

is isomorphic to the trivial deformation $(X, x) \times(S, 0)$ of $(X, x)$. The deformation is said to be locally trivial semiuniversal if it has the usual versality property with respect to locally trivial deformations, i.e. if

$$
X \smile X^{\prime} \longrightarrow\left(S^{\prime}, 0\right)
$$

is any locally trivial deformation of $X$ then there is a cartesian diagram

Communicated by M. Kashiwara, February 17, 1987.

* Sonderforschungsbereich 170 "Geometrie und Analysis", Mathematisches Institut der Universität Bunsenstraße 3/5, D-3400 Göttingen.

** Fach bereich Mathematik der Universität, Universitätsstr. 31, D-8400 Regensburg. 


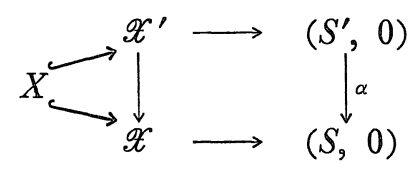

and moreover the tangent map of $\alpha$ in 0 is uniquely determined d $_{\delta}$

In this paper we shall give a positive answer to this question. The basic idea is to reduce it to the following purely local problem. Let

$$
\varphi:(\mathscr{X}, 0) \longrightarrow(S, 0), \quad \phi:(\mathscr{Y}, 0) \longrightarrow(S, 0)
$$

be holomorphic map germs with special fibers $(X, 0) \operatorname{resp} .(Y, 0)$ and

$$
\xi_{0}:(X, 0) \longrightarrow(Y, 0)
$$

be a holomorphic map. A subspace $\left(S^{\prime}, 0\right) \subset(S, 0)$ will be called a maximal extension locus for $\xi_{0}$ if the following two properties are satisfied:

(1) There exists a holomorphic map of $\left(S^{\prime}, 0\right)$-spaces

$$
\xi:\left(\mathscr{X}_{\left.s^{\prime}, 0\right)}^{\longrightarrow}\left(\mathscr{Y}_{s^{\prime}, 0}\right)\right.
$$

extending $\xi_{0}$.

(2) If $\alpha:(T, 0) \longrightarrow(S, 0)$ is a holomorphic map and if there exists a $T \neg$ morphism

$$
\left(\mathscr{X}_{T}, 0\right) \longrightarrow\left(\mathscr{Y}_{T}, 0\right)
$$

extending $\xi_{0}$, then $\alpha$ factorizes over $\left(S^{\prime}, 0\right)$.

In general, such an extension locus will not exist. For instance, let $\mathscr{X}=S$ be the union of two smooth curves $C_{1}, C_{2}$ meeting at 0 with multiplicity $\geq 2$, and let $\mathscr{Y}$ be the union of these two curves meeting transversally at 0
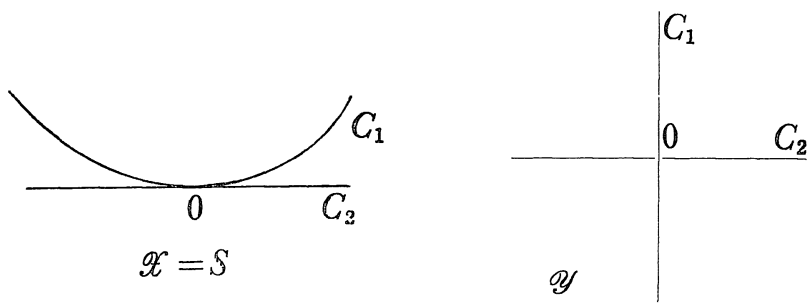

There is a canonical map $\alpha: \mathscr{Y} \longrightarrow S$ and also $\xi_{0}: X=\{0\} \longrightarrow \alpha^{-1}(0)$. This map can be extended over $C_{1}$ and over $C_{2}$ separately as an 
$S$-map but not over the union $S=C_{1} \cup C_{2}$ as the reader may easily verify. What we can prove is the following

(10.1) Theorem. Assume that $\varphi$ above is flat and that the canonical homomorphism

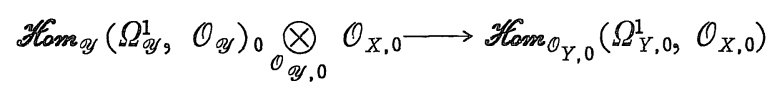

is surjective. Then there exists a maximal extension locus for $\xi_{0}$.

For applications, the most important case is when $\mathscr{Y}=X \times S$ and $\xi_{0}=\mathrm{id}_{X}$. Here the surjectivity condition of $(0,1)$ is automatically satisfied and so we obtain in particular

(0.2) Corollary. If $(\mathscr{X}, 0) \longrightarrow(S, 0)$ is a deformation of $(X, 0)$ then there exists a maximal subspace $\left(S^{\prime}, 0\right) \subset(S, 0)$ for which $\left(\mathscr{X}_{S^{\prime}}, 0\right) \longrightarrow$ $\left(S^{\prime}, 0\right)$ is the trivial deformation, i.e. if $\alpha:(T, 0) \longrightarrow(S, 0)$ is a morphism then

$$
\left(\mathscr{X}_{T}, 0\right) \longrightarrow(T, 0)
$$

is the trivial deformation of $(X, 0)$ iff $\alpha$ factorizes over $\left(S^{\prime}, 0\right)$.

A subspace as in (0.2) will be called the trivial locus of the deformation $(\mathscr{X}, 0) \longrightarrow(S, 0)$.

We remark that $(0.2)$ is easy to prove in the case where $(X, 0)$ has an isolated singularity or, more generally, admits a finite dimensional versal deformation $\left(\mathscr{X}^{*}, 0\right) \longrightarrow\left(S^{*}, 0\right)$. Here the trivial locus of the deformation $(\mathscr{X}, 0) \longrightarrow(S, 0)$ is just $\alpha^{-1}(0)$, if $(\mathscr{X}, 0)$ is obtained from the versal deformation by base change $\alpha:(S, 0) \longrightarrow\left(S^{*}, 0\right)$. But for the general case of $(0.2)$ or $(0.1)$ we must employ Banach analytic methods.

It is easy to see that $(0.2)$ answers the question of Greuel and Karras.

(0.3) Corollary. Any compact complex space $X$ admits a locally trivial semiuniversal deformation. 
Proof. By [Dou $],[\mathrm{F}-\mathrm{K}],[\mathrm{Gra}],[\mathrm{Pa}]$ there is a semiuniversal deformation $\mathscr{X} \rightarrow(S, 0)$ of $X$. For any point $x \in X$ there exists the trivial locus $\left(S_{x}, 0\right) \subset(S, 0)$ for the deformation germ $(\mathscr{X}, x) \rightarrow(S, 0)$. The space $S^{\prime}:=\bigcap_{x \in X} S_{x}$ defines again a subgerm of $(S, 0)$, and it is easy to check that $\mathscr{X}_{S^{\prime}} \rightarrow\left(S^{\prime}, 0\right)$ is the locally trivial semiuniversal deformation of $X$.-

We do not know whether these results also hold in the algebraic case. E. g., if in (0.1) $\varphi, \phi$ and $\xi_{0}$ are algebraic maps, it is natural to ask whether the maximal extension locus of $\xi_{0}$ is also given by algebraic equations. In characteristic $p>0$ we even do not know whether the Schlessinger conditions are satisfied.

We remark that in this paper we also obtain similar results for deformations of other types of analytic objects as e.g. for deformations of holomorphic mappings or for coherent modules. In order to have a unified treatment of these cases we introduce the concept of data of structure preserving maps between complex space germs, see (1. 2), and show that the examples (deformations of spaces, modules and holomorphic maps) fit into this terminology, c.fo (1. 3)-(1. 5).

The basic tool in the proof of our main results are the Banach analytic methods developed by A. Douady in [Dou 1 , [Dou $\left.{ }_{2}\right]$ and also the polycylinder spaces and morphism spaces introduced by G. Pourcin [Pou $]$. In $\S 2$ we review the basic material and prove some simple facts used in the sequel. In $\S 3$ we formulate a Banach analytic condition (A3) on the data of structure preserving maps and show how this condition implies the existence of a subspace $\left(S^{\prime}, 0\right) \subset(S, 0)$ as in (0.1). The verification of this condition (A3) in the case of holomorphic mappings is contained in $\S 4$. The most difficult part there is the proof of the so-called smoothness condition in (A3). In $\S 5$ we give some applications to the case of holomorphic mappings and to modules. In particular, these results imply $(0.1),(0.2)$, (0.3). The first appendix ( $\$ 6)$ contains the notion of a Banach fiber space and we prove there some technical results which are used in this paper and for which there seems to be no reference. Moreover in the second appendix (§7) we give a cohomological description of 
extensions of holomorphic maps which is needed for the examples in $\S 1$ 。

We are going to introduce some notations at first.

Notations. Let $f: X \rightarrow Y$ be a morphism of complex spaces and $\mathscr{M}$ resp. $\mathscr{N}$ be an $\mathcal{O}_{X}$ resp. $\mathcal{O}_{Y}$-module together with a homomorphism

$$
f^{*}(\mathscr{N}) \longrightarrow \mathscr{M}
$$

Then we call $(\mathscr{M}, \mathscr{N})$ an $f$-module. The morphisms of $f$-modules are defined in an obvious way. As it is shown in [FI] $\S 3$, the category of $f$-modules has enough injectives, and so for a complex $\left(\mathscr{K}^{\circ}, \mathscr{L}^{\circ}\right)$ of $f$-modules one can define the Ext-functors in the usual way. These groups will be denoted by

$$
\operatorname{Ext}_{(X, Y)}^{i}\left(\left(\mathscr{K}^{\circ}, \mathscr{L}^{*}\right),(\mathscr{M}, \mathscr{N})\right) \text {. }
$$

If $\mathscr{K}^{\cdot}=\mathscr{K}, \mathscr{L}^{\cdot}=\mathscr{L}$ are modules, then the group $\operatorname{Ext}_{(X, Y)}^{0}((\mathscr{K}, \mathscr{L})$, $(\mathscr{M}, \mathscr{N}))$ is just

$$
\operatorname{Hom}_{(X, Y)}((\mathscr{K}, \mathscr{L}),(\mathscr{M}, \mathscr{N})),
$$

which is the set of all morphisms of $f$-modules.

We can associate to these Ext-groups the $\mathscr{E}_{x t}$-sheaves

$$
\mathscr{E}_{x t_{(X, Y)}^{i}}\left(\left(\mathscr{K}^{\circ}, \mathscr{L}^{\circ}\right),(\mathscr{M}, \mathscr{N})\right)
$$

on $Y$ which are defined by the presheaves

$$
Y \supset V \longmapsto \operatorname{Ext}_{\left(f^{-1}(V), V\right)}^{i}\left(\left.\left(\mathscr{K} \cdot \mathscr{L}^{\cdot}\right)\right|_{V},\left.(\mathscr{M}, \mathscr{N})\right|_{V}\right) \text {. }
$$

If we take the cotangent complexes $\mathscr{K}^{\circ}:=L_{X / Z}^{\circ}, \mathscr{L}^{\circ}:=L_{Y / Z}^{\circ}$, where $\mathrm{Y} \rightarrow \mathrm{Z}$ is a morphism of complex spaces, then the $\mathrm{Ext}^{i}$ resp. $\mathscr{E}_{x \rightarrow} t^{i}$ are also denoted by

$$
T^{i}(f / Z ; \mathscr{M}, \mathscr{N}) \operatorname{resp.} \mathscr{T}^{i}(f / Z ; \mathscr{M}, \mathscr{N}) \text {. }
$$

These invariants are the tangent functors for holomorphic mappings as introduced in $[\mathrm{Fl}]$.

Let $x$ resp. $y$ be a point of $X$ resp. $Y$. Then as usual the stalk of the sheaf

$$
\mathscr{E}_{\mathscr{E}} \dot{t}_{(X, Y)}^{i}\left(\left(\mathscr{K}^{\bullet}, \mathscr{L} \cdot\right),(\mathscr{M}, \mathscr{N})\right)
$$

on $Y$ is denoted by 


$$
\mathscr{E}_{x t_{(X, Y)}^{i}}\left(\left(\mathscr{K}^{\bullet}, \mathscr{L}^{\bullet}\right),(\mathscr{M}, \mathscr{N})\right)_{y}
$$

whereas

$$
\begin{aligned}
& \mathscr{E}_{x t_{(X, Y)}^{i}}\left(\left(\mathscr{K}^{*}, \mathscr{L}^{*}\right),(\mathscr{M}, \mathscr{N})_{(x, y)}:=\right. \\
& \quad \underset{x \in U}{\lim } \mathscr{E}_{x t_{(U, Y)}^{i}}^{i}\left(\left(\mathscr{K} \cdot \mid U, \mathscr{L}^{*}\right),(\mathscr{M} \mid U, \mathscr{N})\right)_{y}
\end{aligned}
$$

and the limit is taken over all open neighborhoods $U$ of $x$ in $X$. In the case $\mathscr{K}^{\bullet}=L_{X / Z}, \mathscr{L}^{\bullet}=L_{Y / Z}$ we write shortly

$$
\mathscr{T}^{i}(f / Z ; \mathscr{M}, \mathscr{N})_{(x, y)} \text {. }
$$

\section{\&1. Data of Structure Preserving Maps}

(1.1) Let $(S, 0)$ be a germ of a complex space and suppose that $\mathscr{X} \rightarrow S$ resp. $\mathscr{Y} \rightarrow S$ are holomorphic maps with special fibres $X$ resp. $Y$. Furthermore we fix a distinguished point 0 in $X$ and $Y$ together with a morphism $\xi_{0}:(X, 0) \rightarrow(Y, 0)$. For any complex space $T \rightarrow S$ let $\mathscr{X}_{T}$ resp. $\mathscr{Y}_{T}$ be the fibre product $T \times_{S} \mathscr{X}$ resp. $T \times{ }_{S} \mathscr{Y}$, and denote by

$$
\operatorname{MDr}_{T}\left(\mathscr{X}_{T}, \mathscr{Y}_{T}\right)
$$

the sheaf on $\mathscr{X}_{T}$ of $T$-morphisms from $\mathscr{X}_{T}$ to $\mathscr{Y}_{T}$, i. e. for an open set $U \subset \mathscr{X}_{T}$ we have

$$
\mathfrak{M D r}_{T}\left(\mathscr{X}_{T}, \mathscr{Y}_{T}\right)(U)=\left\{\varphi: U \longrightarrow \mathscr{Y}_{T} \mid f \text { is a } T \text {-morphism }\right\} .
$$

We shall assume that for every complex space $T$ over $S$, there is given a subsheaf of sets

$$
\mathfrak{M}_{T} \subset \mathfrak{M O r}_{T}\left(\mathscr{X}_{T}, \mathscr{Y}_{T}\right)
$$

such that the following properties are satisfied.

(A 0)

$$
\xi_{0} \in \mathfrak{M}_{\{0\}}(X) \text {, }
$$

(A 1) (Compatibility with fibre products): If $\varphi \in \mathfrak{M}_{T}(U)$ and $T^{\prime} \rightarrow T$ is an $S$-morphism of complex spaces, then

$$
T^{\prime} \times_{T} \varphi \in \mathfrak{M}_{T^{\prime}}\left(T^{\prime} \times_{T} U\right) \text {. }
$$

(A 2) (Extension property). There is a relatively compact neighbourhood $U$ of $O \in X$ with the following property: Let $T^{\prime} \subset T$ be a small extension of Artinian complex space germs over $(S, 0)$, i. e. $\operatorname{Ker}\left(\mathcal{O}_{T, 0} \rightarrow \mathcal{O}_{T^{\prime}, 0}\right)$ is a 1 -dimensional $C$-vectorspace. Suppose that

$$
\varphi:\left(\mathscr{X}_{T}, 0\right) \longrightarrow\left(\mathscr{Y}_{T}, 0\right)
$$


is a germ in $\mathfrak{M}_{T, 0}$ inducing $\xi_{0}$ on the special fibre and that $T^{\prime} \times_{T} \varphi$ is defined over $U$, i. e. comes from a section $\psi^{\prime} \in \mathfrak{M}_{T^{\prime}}(U)$. Then $\psi^{\prime}$ can be extended to a section $\psi \in \mathfrak{M}_{T}(U), i_{\circ}$ e. there is a commutative diagram

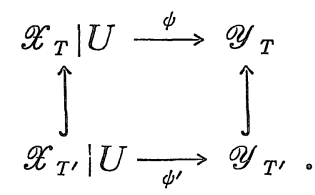

(1.2) Definition. A collection $\mathfrak{M}_{T}, T \in \mathfrak{A} \mathfrak{n} / S$, satisfying (A0)-(A2) is called a datum of structure preserving maps.

In order to clarify what we have in mind, we list a few examples.

(1.3) Example。 Let $(\mathscr{X}, 0),(\mathscr{Y}, 0),(\mathscr{Z}, 0)$ be space germs over the germ $(S, 0)$ and $(\mathscr{X}, 0) \rightarrow(\mathscr{Z}, 0),(\mathscr{Y}, 0) \rightarrow(\mathscr{Z}, 0)$ be $S$-morphisms. Suppose that $\mathscr{X}$ is $S$-flat and $\xi_{0}:(X, 0) \rightarrow(Y, 0)$ is a $\mathscr{Z}$-morphism. Then

$$
\mathfrak{M}_{T}:=\operatorname{Mor}_{T}^{\mathscr{P}}\left(\mathscr{X}_{T}, \mathscr{Y}_{T}\right)
$$

is a datum of structure preserving maps. Here $\mathfrak{M P o r}_{T}^{\mathscr{g}^{g}}(\ldots)$ denotes the subsheaf of $\mathfrak{M l o x}_{T}(\ldots)$ consisting of all $\mathscr{Z}$-morphisms。

Proof. The properties (A0) and (Al) are trivial。 In order to show (A2) let $T, T^{\prime}, \varphi, \varphi^{\prime}:=T^{\prime} \times_{T} \varphi, \psi^{\prime}$ be as in (A2). It follows from $\S 7$ that the obstruction for extending $\psi^{\prime}$ to a $\psi$ lies in

$$
\operatorname{Ext}_{U}^{1}\left(L \xi_{0}^{*}\left(L_{\mathscr{Y} / \mathscr{L}} \stackrel{\otimes}{=} \mathscr{O}_{\mathscr{Y}} \mathcal{O}_{Y}\right), \mathcal{O}_{X}\right),
$$

which for a Stein-neighbourhood is just $\Gamma(U, \mathscr{E})$ where

$$
\mathscr{E}:=\mathscr{E} x t_{X}^{1}\left(L \xi_{0}^{*}\left(L_{\mathscr{O} / \mathscr{L}}^{\circ} \stackrel{\otimes}{=} \mathscr{O}_{\mathscr{Y}} \mathcal{O}_{Y}\right), \mathcal{O}_{X}\right) \text {. }
$$

On the other hand, the obstruction for extending $\varphi^{\prime}$ to $\varphi$ is in the stalk $\mathscr{E}_{0}$. Since for a privileged neighbourhood $U$ of 0 (for instance in the sense of $[B-\mathbb{K}]$ (II 3.9)) the restriction map

$$
\Gamma(U, \mathscr{E}) \longrightarrow \mathscr{E}_{0}
$$

is injective, see loc。 cit (II 3.10), (A2) follows.- 
(1.4) Example. Let

$$
\xi:(\mathscr{X}, 0) \longrightarrow(\mathscr{Y}, 0)
$$

be a morphism of complex space germs over the germ $(S, 0)$ with fibres $X \subset \mathscr{X}$ resp. $Y \subset \mathscr{Y}$ over $0 \in S$. Moreover we fix a coherent $\mathcal{O}_{\mathscr{X}}$ resp. $\mathcal{O}_{\mathscr{y}}$-module $\mathscr{F}$ resp. $\mathscr{G}$ and a homomorphism

$$
\varphi_{0}: \xi_{0}^{*}\left(\mathscr{G}_{0}\right) \longrightarrow \mathscr{F}_{0}
$$

where the index " 0 " indicates the restriction to the special fibre. We shall always assume that $\mathscr{F}$ is $S$-flat (but $\mathscr{G}$ may be arbitrary). Now we consider the infinitesimal extensions

$$
\mathscr{X}^{\prime}:=\left(|\mathscr{X}|, \mathcal{O}_{\mathscr{X}}[\mathscr{F}]\right), \quad \mathscr{Y}^{\prime}:=\left(|\mathscr{Y}|, \mathcal{O}_{\mathscr{Y}}[\mathscr{G}]\right)
$$

and define for any morphism $T \rightarrow S$ the sheaf

$$
\mathfrak{M}_{T} \subset \mathfrak{M o r}_{T}\left(\mathscr{X}_{T}^{\prime}, \mathscr{Y}_{T}^{\prime}\right)
$$

of all morphisms which preserve the grading and induce the given map $\xi_{T}$ on $\mathscr{X}_{T} \subset \mathscr{X}_{T}^{\prime}$. Obviously the collection $\mathfrak{M}_{T}$ satisfies (A0) with $\left(\xi_{0}, \varphi_{0}\right)$ and (Al). Moreover, the sheaf $\mathfrak{M}_{T}$ is canonically isomorphic to the sheaf of homomorphisms of modules

$$
\mathfrak{M}_{T}(U)=\operatorname{Hom}_{U}\left(\left(\xi_{T} \mid U\right) *\left(\mathscr{G}_{T}\right), \mathscr{F}_{T} \mid U\right)
$$

for $U \subset \mathscr{X}_{T}$ open.

It is well known that, in the situation of (A2), the obstruction for extending a homomorphism

$$
\xi_{T^{\prime}}^{*}\left(\mathscr{G}_{T^{\prime}}\right) \longrightarrow \mathscr{F}_{T^{\prime}}
$$

to a homomorphism $\xi_{T}^{*}\left(\mathscr{G}_{T}\right) \rightarrow \mathscr{F}_{T}$ lies in

$$
\operatorname{Ext}_{X}^{1}\left(L \xi_{0}^{*}\left(\mathscr{G} \stackrel{\otimes}{=}{ }_{\mathscr{O}} \mathcal{O}_{Y}\right), \mathscr{F}_{0}\right) .
$$

So with a similar argument as in the proof of (1.3), one sees that (A2) is satisfied and $\left(\mathfrak{M}_{T}\right)_{T}$ is a datum of structure preserving maps.

(1.5) Example. Let

$$
\begin{aligned}
& \left(\mathscr{X}_{0}, 0\right) \stackrel{f}{\longrightarrow}\left(\mathscr{X}_{1}, 0\right) \longrightarrow \\
& \left(\mathscr{Y}_{0}, 0\right) \underset{g}{\longrightarrow}\left(\mathscr{Y}_{1}, 0\right) \longrightarrow
\end{aligned}(\mathscr{Z}, 0) \longrightarrow(S, 0)
$$


be a diagram of morphisms of complex space germs over $(S, 0)$ and let $\left(X_{0}, 0\right),\left(X_{1}, 0\right),\left(Y_{0}, 0\right),\left(Y_{1}, 0\right),(Z, 0), f_{0}, g_{0}$ be the respective fibres over $0 \in S$. We assume that there are given morphisms $\xi_{i}:\left(X_{i}, 0\right)$ $\rightarrow\left(Y_{i}, 0\right), i=0,1$ such that

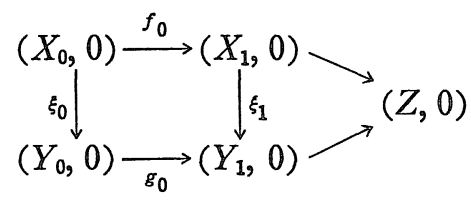

commutes. We put

$$
\mathscr{X}:=\mathscr{X}_{0} \times_{\mathscr{L}} \mathscr{X}_{1}, \quad \mathscr{Y}:=\mathscr{Y}_{0} \times_{\mathscr{L}} \mathscr{Y}_{1}
$$

and consider the subsheaf

$$
\mathfrak{M}_{T} \subset \mathfrak{M n x}_{T}^{\mathscr{P}}\left(\mathscr{X}_{T}, \mathscr{Y}_{T}\right)
$$

consisting locally in $\mathscr{X}_{T}$ of all $T$-morphisms $\zeta=\zeta_{0} \times \zeta_{1}: \mathscr{X}_{T} \rightarrow \mathscr{Y}_{T}$ where $\zeta_{i}: \mathscr{X}_{i T} \rightarrow \mathscr{Y}_{i T}$ is a $\mathscr{Z}_{T}$-morphism such that $\zeta$ maps the graph of $f_{T}$ into the graph of $g_{T}$. Equivalently, a local section of $\mathfrak{M}_{T}$ around a point of graph $\left(f_{T}\right)$ may be regarded as a commuting diagram (of germs)

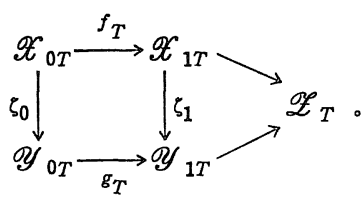

Then $\left(\mathfrak{M}_{T}\right)_{T}$ is a datum of structure preserving maps with $\left(\xi_{0}, \xi_{1}\right)$ in the special fibre, if the following conditions are satisfied

(i) $\mathscr{X}_{0}, \mathscr{X}_{1}$ are $S$-flat,

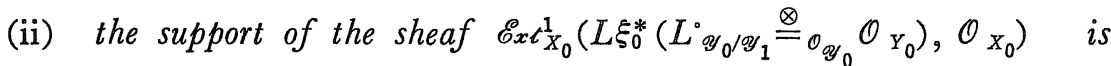
finite over $X_{1}$.

Proof. Condition (A0) is satisfied with $\left(\xi_{0}, \xi_{1}\right)$ and (A1) is trivial. We have to verify (A2)。 Let $T^{\prime} \longrightarrow T$ be as in (A2) and suppose that there is a structure preserving map

$$
\left(\zeta_{0}, \zeta_{1}\right): \mathscr{X}_{{ }_{0 T}} \times_{\mathscr{T}_{T}}, \mathscr{X}_{1 T^{\prime}} \longrightarrow \mathscr{Y}_{{ }_{0 T}} \times_{\mathscr{X}_{T}}, \mathscr{Y}_{1 T^{\prime}} \text { inducing }\left(\xi_{0}, \xi_{1}\right)
$$

in the special fibre. Furthermore, we denote by $\zeta_{i}^{\prime}$ the composition $\mathscr{X}_{i T} \longrightarrow \mathscr{Y}_{i T}, \stackrel{p r}{\longrightarrow} \mathscr{Y}_{i}$ for $i=0,1$. Then, according to $\S 7$, the obstruction for extending $\left(\zeta_{0}, \zeta_{1}\right)$ to a structure preserving map 


$$
\left(\eta_{0}, \eta_{1}\right): \mathscr{X}_{0 T} \times_{\mathscr{Z}_{T}} \mathscr{X}_{1 T} \longrightarrow \mathscr{Y}_{0 T} \times_{\mathscr{Z}_{T}} \mathscr{Y}_{1 T}
$$

lies in the group

$$
E^{1}:=\operatorname{Ext}_{\left(\mathscr{X}_{0 T}, \mathscr{X}_{1 T^{\prime}}\right)}\left(\left(L \zeta_{0}^{\prime *}\left(L_{\mathscr{O}_{0} / \mathscr{Z}}\right), L \zeta_{1}^{\prime *}\left(L_{\mathscr{\mathscr { V }}_{1} / \mathscr{L}}\right)\right),\left(\mathcal{O}_{X_{0}}, \mathcal{O}_{X_{1}}\right)\right) .
$$

For abbreviation we set

$$
\begin{aligned}
& \mathscr{E}_{i}^{:}:=L \zeta_{i}^{\prime *}\left(L_{\mathscr{\mathscr { Y }}_{i / \mathscr{L}}}\right), \quad i=0,1 \\
& \mathscr{E}_{i}:=L \zeta_{0}^{\prime *}\left(L_{\mathscr{Y}_{0} / \mathscr{Y}_{1}}\right)
\end{aligned}
$$

and get a short exact sequence

$$
0 \longrightarrow L f_{T^{\prime}}^{*}\left(\mathscr{E}_{i}^{i}\right) \longrightarrow \mathscr{E}_{0} \longrightarrow \mathscr{E}_{i} \longrightarrow 0 .
$$

Defining

$$
\begin{aligned}
& \overline{\mathscr{E}}_{i}:=\mathcal{O}_{X_{i}} \stackrel{\otimes}{=} \mathscr{O}_{\mathscr{X}_{i}} \mathscr{E}_{i}, \quad i=0,1 \\
& \overline{\mathscr{E}}_{2}:=\mathcal{O}_{X_{0}} \stackrel{\otimes}{=} \mathscr{O}_{\mathscr{X}_{0}} \mathscr{E} \dot{E}_{2}
\end{aligned}
$$

we get again a short exact sequence

$$
0 \longrightarrow L f_{0}^{*}\left(\overline{\mathscr{E}}_{i}\right) \longrightarrow \overline{\mathscr{E}}_{0} \longrightarrow \overline{\mathscr{E}}_{2} \longrightarrow 0 \text { 。 }
$$

Obviously we have

$$
E^{1}=\operatorname{Ext}_{\left(X_{0}, X_{1}\right)}^{1}\left(\left(\overline{\mathscr{E}}_{0}, \overline{\mathscr{E}}_{i}\right),\left(\mathcal{O}_{X_{0}}, \mathcal{O}_{X_{1}}\right)\right) .
$$

Similarly as before, we want to show that for suitable neighbourhoods $U$ and $V$ of $0 \in X_{0}$ resp. $0 \in X_{1}$ with $f_{0}(U) \subset V$, the canonical map

$$
\begin{aligned}
\operatorname{Ext}_{(U, V)}^{1}\left(\left(\overline{\mathscr{E}}_{0}, \overline{\mathscr{E}}_{i}\right),\left(\mathcal{O}_{X_{0}}, \mathcal{O}_{X_{1}}\right)\right) \longrightarrow \\
\mathscr{E}_{x t_{\left(X_{0}, X_{1}\right)}^{1}}\left(\left(\overline{\mathscr{E}}_{0} \dot{\mathscr{E}}_{i}\right),\left(\overline{\mathscr{E}}_{X_{0}}, \mathcal{O}_{X_{1}}\right)\right)_{(0,0)}
\end{aligned}
$$

is injective. By $[\mathrm{Fl}](3.5),[\mathrm{B}-\mathrm{K}](\mathbb{I} 12.12)$ and the data above, we have an exact sequence of sheaves on $X_{1}$

$$
\begin{aligned}
& \cdots \longrightarrow \mathscr{H}_{0 m}\left(\overline{\mathscr{E}}_{1}, \mathcal{O}_{X_{1}}\right) \stackrel{\delta}{\longrightarrow} f_{0 *}\left(\mathscr{E}_{x t} t^{1}\left(\overline{\mathscr{E}}_{2}, \mathcal{O}_{X_{0}}\right)\right) \longrightarrow \\
& \mathscr{E}_{x} t^{1}\left(\left(\overline{\mathscr{E}}_{0}, \overline{\mathscr{E}}_{\mathrm{i}}\right),\left(\mathcal{O}_{X_{0}}, \mathcal{O}_{X_{1}}\right)\right) \longrightarrow \mathscr{E}_{x} \iota^{1}\left(\overline{\mathscr{E}}_{i}, \mathcal{O}_{X_{1}}\right) \longrightarrow \\
& f_{0 *}\left(\mathscr{E}_{x} \iota^{2}\left(\overline{\mathscr{E}}_{2}, \mathcal{O}_{X_{0}}\right)\right) \longrightarrow \ldots
\end{aligned}
$$

From assumption (ii) follows that Coker $(\delta)$ is a coherent $\mathcal{O}_{X_{1}}$-module. Hence, if we take $V$ privileged for $\operatorname{Coker}(\delta)$ and $\mathscr{E}_{x} t^{1}\left(\overline{\mathscr{E}}_{1}, \mathcal{O}_{X_{1}}\right)$, then $(*)$ is injective.- 
(1.6) Let now $\mathscr{X} \rightarrow S, \mathscr{Y} \rightarrow S$ and $\xi_{0}:(X, 0) \rightarrow(Y, 0)$ be as at the beginning of this section and let

$$
\mathfrak{M}_{T} \subset \mathfrak{M D r}_{T}\left(\mathscr{X}_{T}, \mathscr{Y}_{T}\right), \quad T \in \mathfrak{U} \mathfrak{n} / S
$$

be a datum of structure preserving maps. We consider the following functor on germs of complex spaces over $(S, 0)$ :

$$
F: \mathfrak{U} \mathfrak{H} /(S, 0) \longrightarrow \mathfrak{G} \text { et }
$$

where

$$
\begin{aligned}
& F((T, 0))=\{1\}, \text { if there is a local section } \\
& \varphi:\left(\mathscr{X}_{T}, 0\right) \rightarrow \mathscr{Y}_{T} \text { in } \mathfrak{M}_{T} \text { which induces } \xi_{0} \\
& \text { on the special fibre }
\end{aligned}
$$

and

$$
F((T, 0))=\emptyset \text { otherwise. }
$$

If $F$ is representable by the $(S, 0)$-germ $\left(S^{\prime}, 0\right)$ then we call $\left(S^{\prime}, 0\right)$ the maximal extension locus of $\xi_{0}$. Observe that such an $\left(S^{\prime}, 0\right)$ is automatically a subspace of $(S, 0)$.

In general, a maximal extension subspace need not exists as was shown by the example mentioned in the introduction. In Section 3 we shall give a criterion for the representability of the functor $F$. In the proof we will construct arbitrary small compact neighbourhoods $K$ of $0 \in X$ and closed subgerms $\left(S_{K}, 0\right) \subset(S, 0)$ with the following properties:

(1.7) (1) Let $\alpha:(T, 0) \rightarrow(S, 0)$ be a morphism and suppose that $\varphi \in \mathfrak{M}_{T}(K)$ is a morphism extending $\xi_{0}$ which is defined in a neighbourhood of $K \longrightarrow X \longrightarrow \mathscr{X}_{T}$. Then $\alpha$ factorizes via $\left(S_{K}, 0\right)$.

(2) Let $\left(T^{\prime}, 0\right) \longrightarrow(T, 0) \subset\left(S_{K}, 0\right)$ be closed complex subspaces and assume that $\varphi^{\prime} \in \mathfrak{M}_{T^{\prime}}(K)$ is a morphism extending $\xi_{0}$. Then there is a $\varphi \in \mathfrak{M}_{T}\left(K^{\circ}\right)$, such that $T^{\prime} \times_{T} \varphi$ and $\varphi^{\prime}$ coincide on $\stackrel{\circ}{K}$.

We shortly call such a neighbourhood $K$ a good neighbourhood of 0 . If $L \subset \stackrel{\circ}{K}$ are good neighbourhoods then, by the properties (1), (2) above, $S_{K}$ is a subspace of $S_{L}$.

(1.8) Proposition. Assume that there is a basis of good neighbourhoods of $0 \in X$. Then the functor $F$ above is representable by a closed subgerm 
$\left(S^{\prime}, 0\right) \subset(S, 0)$. Moreover, $\left(S^{\prime}, 0\right)=\left(S_{K}, 0\right)$ for every sufficientlv small good neighbourhood $K$ of 0 .

Proof. Let $U$ be a neighbourhood as in (A2). We show that for good neighbourhoods $L \subset K \subset U$ we have $\left(S_{L}, 0\right)=\left(S_{K}, 0\right)$. For this it is sufficient to prove that for every artinian subspace $(T, 0)$ contained in $\left(S_{L}, 0\right)$, there is a morphism $\varphi \in \mathfrak{M}_{T}(U)$. By induction we may assume that there exists a complex subspace $T^{\prime} \subset T$ such that $T^{\prime} \longleftrightarrow T$ is a small extension and that there is already a morphism

$$
\varphi^{\prime} \in \mathfrak{M}_{T^{\prime}}(U) \text {. }
$$

Using property (1.7) (2), we can extend $\varphi^{\prime}$ to a morphism $\tilde{\varphi} \in \mathfrak{M}_{T}(\stackrel{\circ}{L})$, i. e. $T^{\prime} \times_{T} \tilde{\varphi}$ and $\varphi^{\prime}$ coincide on $\stackrel{\circ}{L}$. Now (A2) implies that $\varphi^{\prime}$ can also be extended to a $\varphi \in \mathfrak{M}_{T}(U) .-$

\section{§2. Polycylinder Spaces and Morphism Spaces}

In this section we review some more or less known facts about the polycylinder and morphism spaces introduced by Pourcin in [Pou $]$.

Let $K=K_{1} \times \ldots \times K_{n} \subset \mathbb{C}^{n}$ be a polycylider, i. e. each $K_{i} \subset \mathbb{C}$ is compact and convex with $\stackrel{\circ}{K}_{i} \neq \phi$. We denote by $B(K)$ the Banach algebra of continuous $\mathbb{C}$-valued functions on $K$ which are holomorphic on $\stackrel{\circ}{\text {. }}$

If $\mathscr{F}$ is a coherent sheaf in a neighbourhood of $K$, then $\mathscr{F}$ is called $K$-privileged (see $\left[\mathrm{Dou}_{1}\right]$ Chap. 7 ) if there is a resolution

$$
0 \longrightarrow \mathcal{O}_{K}^{k} \longrightarrow \cdots \longrightarrow \mathcal{O}_{K}^{k} \longrightarrow \mathscr{F} \mid K \longrightarrow 0
$$

such that the sequence

$$
B\left(K, \mathcal{O}_{\dot{K}}^{k}\right)
$$

of Banach spaces is direct exact. In this situation $B(K, \mathscr{F})$ denotes the cokernel of

$$
B\left(K, \mathcal{O}_{K}^{k_{1}}\right) \longrightarrow B\left(K, \mathcal{O}_{K}^{k_{0}}\right)
$$

It is well known that this splitting property as well as the construction of $B(K, \mathscr{F})$ do not depend on the chosen resolution, cf. [Dou $]$.

In the following we shall need the polycylinder spaces as intro- 
duced by Pourcin $\left[\mathrm{Pou}_{1}\right]$. Let $\mathscr{B}_{K}$ be the sheaf of all continuous functions on $K$ which are holomorphic in the interior, i. e.

$$
\mathscr{B}_{K}(U)=\{f: U \rightarrow \mathbb{C} \mid f \text { continuous, } f \mid \stackrel{\circ}{K} \cap U \text { holomorphic }\} \text { 。 }
$$

Then $\mathscr{B}_{K}$ is a sheaf for which Theorem A and Theorem B hold, i. e.

$$
\begin{aligned}
& \Gamma\left(K, \mathscr{B}_{K}\right)=B(K), \\
& H^{i}\left(K, \mathscr{B}_{K}\right)=0, \quad \text { for all } i>0 .
\end{aligned}
$$

For any local analytic subspace $X$ of $\mathbb{C}^{n}$ which is closed in some neighbourhood of $K$ and $K$-privileged for $\mathcal{O}_{X}$, there is a ringed space

$$
\left(X_{K}, \mathscr{B}_{X_{K}}\right)
$$

where $X_{K}:=X \cap K$ and $\mathscr{B}_{X_{K}}$ is the quotient of $\mathscr{B}_{K}$ by the defining ideal of $X$. Similarly as above we have

$$
\begin{aligned}
& \Gamma\left(X_{K}, \mathscr{B}_{X_{K}}\right)=B\left(K, \mathcal{O}_{X}\right), \\
& H^{i}\left(X_{K}, \mathscr{B}_{X_{K}}\right)=0, \quad \text { for all } i>0 .
\end{aligned}
$$

We remark that there is even a natural structure of a functored space on $X_{K}$, see $\left[\mathrm{Pou}_{1}\right]$ for details.

We also need a relative version of these polycylinder spaces: Let $S$ be a Banach analytic space and

$$
\mathscr{X} \longleftrightarrow S \times \mathbb{C}^{n}
$$

an $S$-anaflat subspace which is closed in some neighbourhood of $S \times K$. If $\mathscr{X}(s) \subset \mathbb{C}^{n}$ denotes the fibre of $\mathscr{X}$ over $s \in S$, then by a result of Douady the set of points $s$ such that $\mathcal{O}_{\mathscr{C}(s)}$ is $K$-privileged is an open subset of $S$. Assuming that $\mathcal{O}_{\mathscr{X}(s)}$ is $K$-privileged for all $s \in S$, then one can construct a locally trivial bundle of Banach spaces $B\left(K, \mathcal{O}_{\mathscr{X}}\right)$ over $S$ whose fibre over $s \in S$ is just $B\left(K, \mathcal{O}_{\mathscr{X}(s)}\right)$, see [Dou $]$ Chap. 8, No. 3. Moreover one can equip

$$
\mathscr{X}_{K}:=\mathscr{X} \cap(S \times K)
$$

with the structure of a functored space and in particular with a structure sheaf $\mathscr{B}_{\mathscr{X}_{K}}$ such that $\mathscr{X}_{K} \rightarrow S$ is a morphism with fibres $\mathscr{X}(s)_{K}$. In the following we call $\mathscr{X}_{K}$ a polycylinder space over $S$. For any morphism $T \rightarrow S$ of Banach analytic spaces

$$
\mathscr{X}_{K, T}
$$

denotes the polycylinder space $\left(T \times{ }_{s} \mathscr{X}\right) \cap(T \times K)$. For the details 
the reader is referred to $\left[\mathrm{Pou}_{1}\right]$ Chap. 4.

A second important tool are the morphism spaces which we are going to describe now. If $S, \mathscr{X}, \mathscr{X}_{K}$ are as before and $\mathscr{Y} \rightarrow S$ is a space of finite presentation over $S$, then we consider the following functor

$$
\operatorname{Mor}_{S}\left(\mathscr{X}_{K}, \mathscr{Y}\right): \mathfrak{B a n} / S \longrightarrow \text { get? }
$$

on the category of Banach analytic spaces over $S$, given by

$$
\underline{\operatorname{Mor}_{S}}\left(\mathscr{X}_{K}, \mathscr{Y}\right)(T):=\operatorname{Mor}_{T}\left(\mathscr{X}_{K, T}, \mathscr{Y}_{T}\right) \text {, }
$$

where $\operatorname{Mor}_{T}\left(\mathscr{X}_{K, T}, \mathscr{Y}_{T}\right)$ is the set of all $T$-morphisms from $\mathscr{X}_{K, T}$ into $\mathscr{Y}_{T}$ of functored spaces.

(2.1) Theorem (Pourcin). The functor $\operatorname{Mor}_{S}\left(\mathscr{X}_{K}, \mathscr{Y}\right)$ is representable by a Banach analytic space $M_{S}\left(\mathscr{X}_{K}, \mathscr{Y}\right)$ over $S$.

The underlying set of the fibre of $M_{S}\left(\mathscr{X}_{K}, \mathscr{Y}\right)$ in a point $s \in S$ consists of all morphisms $g$ of $\mathscr{X}_{K}(s)$ into $\mathscr{Y}(s)$. The point in $M_{S}\left(\mathscr{X}_{K}, \mathscr{Y}\right)$ associated to $g$ and $s$ will be denoted by $(s, g)$ in the following. If $S$ is reduced to a point we simply write Mor(...), $M(\ldots)$ instead of $\operatorname{Mor}_{S}(\ldots), M_{S}(\ldots)$.

Now assume that $\mathscr{Z}$ is another space of finite presentation over $S$ and that there are $S$-morphisms

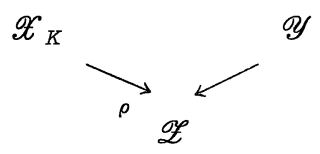

Then we denote by $\operatorname{Mor}_{2}^{\mathscr{Z}}(\ldots)$ the set of all $\mathscr{Z}$-morphisms.

(2.2) Proposition. The functor

$$
T \longmapsto \operatorname{Mor}_{T}^{\mathscr{Z}}\left(\mathscr{X}_{K, T}, \mathscr{Y}_{T}\right),
$$

defined on $\mathfrak{B a n} / S$ is representable by a Banach analytic subspace

$$
M_{S}^{\mathscr{F}}\left(\mathscr{X}_{K}, \mathscr{Y}\right) \subset M_{S}\left(\mathscr{X}_{K}, \mathscr{Y}\right) \text {. }
$$

Proof. Composing with the map $\mathscr{Y} \rightarrow \mathscr{Z}$ gives a morphism

$$
M_{S}\left(\mathscr{X}_{K}, \mathscr{Y}\right) \longrightarrow M_{S}\left(\mathscr{X}_{K}, \mathscr{Z}\right) \text {, }
$$


and the given $S$-map $\mathscr{X}_{K} \rightarrow \mathscr{Z}$ induces a section $S \rightarrow M_{S}\left(\mathscr{X}_{K}, \mathscr{Z}\right)$. Obviously, the space

$$
M_{S}^{\mathscr{F}}\left(\mathscr{X}_{K}, \mathscr{Y}\right):=S \times_{M_{S}\left(\mathscr{X}_{K}, \mathscr{Z}\right)} M_{S}\left(\mathscr{X}_{K}, \mathscr{Y}\right)
$$

represents the functor above.-

It will be important for us in the later sections to have a rather explicite description of the relative tangent space of

$$
M:=M_{S}^{\mathscr{F}}\left(\mathscr{X}_{K}, \mathscr{Y}\right) \text {. }
$$

For our purposes it is sufficient to restrict ourselves to the case where $S$ is finite dimensional and where

$$
\mathscr{Y} \subset U \subset \mathscr{Z} \times \mathbb{C}^{p}
$$

is a closed complex subspace of the Stein open set $U$ in $\mathscr{Z} \times \mathscr{C}^{p}$ given by the equations $f=\left(f_{1}, \ldots, f_{q}\right): U \rightarrow \mathbb{C}^{q}$.

If $\mathscr{E}$ is a coherent sheaf on $\mathscr{Y}$ having a presentation

$$
\mathcal{O}_{\mathscr{Y}}^{q} \stackrel{g}{\longrightarrow} \mathcal{O}_{\mathscr{Y}}^{p} \longrightarrow \mathscr{E} \longrightarrow 0,
$$

then we can associate to $\mathscr{E}$ the Banach fibre space (in the sense of §)

$$
\mathbb{V}(M ; \mathscr{E}):=\operatorname{Ker}\left(M \times{ }_{S} B\left(K, \mathcal{O}_{\mathscr{X}}^{p}\right) \stackrel{\tilde{g}}{\longrightarrow} M \times{ }_{s} B\left(K, Q_{\mathscr{C}}^{q}\right)\right)
$$

with $\tilde{g}:=\Phi^{*}\left({ }^{t} g\right)$, where

$$
\Phi: \mathscr{X}_{K, M} \longrightarrow \mathscr{Y}
$$

is the universal morphism. It is easy to check that $\mathbb{V}(M ; \mathscr{E})$ is independent of the representation and functorial in $\mathscr{E}$. We claim

(2.3) Proposition. The relative tangent space $T(M / S)$ is canonically isomorphic to the Banach fibre space

$$
\mathbb{V}\left(M ; \Omega_{\mathscr{Y} / \mathscr{L}}^{1}\right) \text {. }
$$

Proof. If $\mathscr{Y}=\mathscr{Z} \times \mathbb{C}^{p}$, then $M \cong B\left(K, \mathcal{O}_{\mathscr{X}}\right)^{p}$ and so $T(M / S)$ is isomorphic to the trivial Banach bundle over $M$

$$
M \times{ }_{S} B\left(K, \mathcal{O}_{\mathscr{C}}^{p}\right) \text { 。 }
$$

Similarly, for any open subset $U \subset \mathscr{Z} \times \mathbb{C}^{p}$ the space $M_{S}^{\mathscr{Z}}\left(\mathscr{X}_{K}, U\right)$ is the open subset of $B\left(K, \mathcal{O}_{\mathscr{C}}^{p}\right)$ consisting of all $\left(s, h_{1}, \ldots, h_{p}\right)$ such that $\left(\rho_{s}, h_{1}, \ldots, h_{p}\right)\left(\mathscr{X}_{K}(s)\right) \subset U$. If now $\mathscr{Y} \subset U$ is given by the 
equations $f_{1}, \ldots, f_{q}$, then $M_{S}\left(\mathscr{X}_{K}, \mathscr{Y}\right)$ is the preimage of the zero section under the morphism

$$
\begin{aligned}
& M_{S}\left(\mathscr{X}_{K}, U\right) \stackrel{F}{\longrightarrow} B\left(K, \mathcal{O}_{\mathscr{Q}}^{q}\right) \\
& \left(s, h_{1}, \ldots, h_{p}\right) \longmapsto\left(s, f_{1} \circ h, \ldots, f_{q} \circ h\right) \text { if } h=\left(h_{1}, \ldots, h_{p}\right) .
\end{aligned}
$$

Hence the tangent space of $M=M_{S}\left(\mathscr{X}_{K}, \mathscr{Y}\right)$ is the kernel of the bundle map

$$
M \times{ }_{S} B\left(K, \mathcal{O}_{\mathscr{X}}^{p}\right) \stackrel{T(F)}{\longrightarrow} M \times{ }_{S} B\left(K, \mathcal{O}_{\mathscr{X}}^{q}\right)
$$

and it is easily seen that $T(F)$ is just given by the matrix of partial derivatives

$$
\frac{\partial f_{i}}{\partial z_{j}}, \quad 1 \leq i \leq q, 1 \leq j \leq p
$$

Using the presentation

$$
\begin{aligned}
\mathcal{O}_{\mathscr{Y}}^{q} & \longrightarrow \mathcal{O} \\
e_{\mathscr{Y}} & \otimes_{\mathcal{O}_{U}} \Omega_{U / \mathscr{Z}}^{1} \longrightarrow 1 \otimes \Omega_{\mathscr{Y} / \mathscr{Z}}^{1} \longrightarrow 0 \\
e_{U / \mathscr{Z}} & \left(f_{i}\right)
\end{aligned}
$$

of $\Omega_{\mathscr{Y} / \mathscr{Z}}^{1}$ the assertion follows.-

We also note

(2.4) Proposition. Let $g: \mathscr{Y}_{1} \rightarrow \mathscr{Y}_{2}$ be a $\mathscr{Z}$-morphism ( $\mathscr{Y}_{i}$ as above) and consider the map induced by $g$

$$
\Gamma_{g}: M_{1} \longrightarrow M_{2}, \quad M_{i}:=M_{S}^{\mathscr{F}}\left(\mathscr{X}_{K}, \mathscr{Y}_{i}\right), \quad i=1,2 .
$$

Then the tangent map $T\left(\Gamma_{g} / S\right)$ of $\Gamma_{g}$ is induced by the canonical map

$$
d g: g^{*}\left(\Omega_{\mathscr{Y}_{2} / \mathscr{Z}}^{1}\right) \longrightarrow \Omega_{\mathscr{Y}_{1} / \mathscr{Z}}^{1} \text {. }
$$

Observe that for coherent sheaves $\mathscr{E}_{i}$ on $\mathscr{Y}_{i}, i=1,2$, admitting global finite presentations, any homomorphism

$$
\alpha: g^{*}\left(\mathscr{E}_{2}\right) \longrightarrow \mathscr{E}_{1}
$$

induces a $\Gamma_{g}$-map

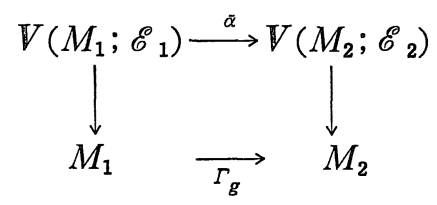


of Banach fibre spaces. With the identification of (2.3), proposition (2.4) says that

$$
T\left(\Gamma_{g} / S\right)=\widetilde{d g}
$$

The proof of (2.4) is straightforward and left to the reader as an exercise.

Later we also need the derivative of the composition mapping between the morphism spaces with respect to the first variable. So let

$$
\begin{aligned}
& \mathscr{X}_{1} \subset S \times \mathbb{C}^{n_{1}^{\prime}}, \\
& \mathscr{X}_{2} \subset S \times \mathbb{C}^{n_{2}}
\end{aligned}
$$

be closed analytic subspaces in some neighbourhoods of the polycylinder spaces $S \times K_{1}^{\prime}$ resp. $S \times K_{2}$ (where $K_{1}^{\prime}$ resp. $K_{2}$ is privileged for $\mathscr{X}_{1}(s)$ resp. $\left.\mathscr{X}_{2}(s), s \in S\right)$ and let $f: \mathscr{X}_{1} \rightarrow \mathscr{X}_{2}$ be a $S$-morphism. We take the product embedding

$$
\mathscr{X}_{1} \longrightarrow S \times \mathbb{C}^{n_{1}}, \quad n_{1}:=n_{1}^{\prime}+n_{2}
$$

so that $f$ is induced by the canonical projection $S \times \mathbb{C}^{n_{1}} \rightarrow S \times \mathbb{C}^{n_{2}}$. If we set $K_{1}:=K_{1}^{\prime} \times K_{2}$ and assume that $\mathcal{O}_{\mathscr{X}_{1}(\mathrm{~s})}$ is $K_{1}$-privileged for all $s \in S$, there is a morphism, also denoted by $f$

$$
\mathscr{X}_{1 K_{1}} \longrightarrow \mathscr{X}_{2 K_{2}}
$$

of functored spaces and so there is an $S$-morphism

$$
{ }_{f} \Gamma: M_{2} \longrightarrow M_{1}, \quad M_{i}:=M_{S}^{\mathscr{F}}\left(\mathscr{X}_{i K_{i}}, \mathscr{Y}\right) \text { 。 }
$$

Let $\Phi_{i}: \mathscr{X}_{i K_{i}, M_{i}} \longrightarrow \mathscr{Y}$ be the universal map for $i=1,2$ and $\mathscr{E}$ a coherent $\mathcal{O}_{\mathscr{Y}}$-module admitting a global finite presentation. The commutative diagram

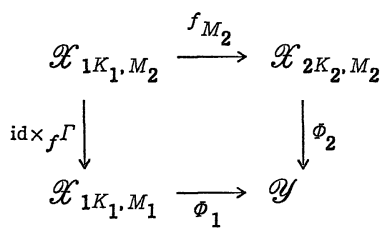

induces a canonical ${ }_{f} \Gamma$-morphism

$$
\tilde{f}(\mathscr{E}): \mathbb{V}\left(M_{2} ; \mathscr{E}\right) \longrightarrow \mathbb{V}\left(M_{1} ; \mathscr{E}\right)
$$

of Banach fibre spaces. We claim

(2.5) Proposition. $\tilde{f}\left(\Omega_{\mathscr{y} / \mathscr{Z}}^{1}\right)$ is the tangent map of ${ }_{f} \Gamma$ 。 
Proof. It is sufficient to consider the case

$$
\mathscr{Y}=\mathscr{Z} \times \mathbb{C} \text {. }
$$

In this situation $M_{i}=B\left(K_{i}, \mathcal{O}_{\mathscr{x}_{i}}\right)$ and we have

$$
\begin{gathered}
{ }_{f} \Gamma: B\left(K_{2}, \mathcal{O}_{\mathscr{C}_{2}}\right) \longrightarrow B\left(K_{1}, \mathcal{O}_{\mathscr{x}_{1}}\right) \\
h \longmapsto h \circ f .
\end{gathered}
$$

Since the map is linear, the tangent map just equals ${ }_{f} \Gamma$ and this shows (2.5).-

\section{§3. The Representability Theorem}

Let $\mathscr{X}, \mathscr{Y}, X, Y, \xi_{0}$ be as in (1.1) and $\mathfrak{M}_{T} \subset \mathfrak{M O r}_{T}\left(\mathscr{X}_{T}, \mathscr{Y}_{T}\right)$ a datum of structure preserving maps, see (1.2). We fix an embedding $i: X \longrightarrow \mathbb{C}^{n}$ and an $S$-embedding $\mathscr{X} \longrightarrow S \times \mathbb{C}^{n}$ which induces $i$ on the special fibre.

For every $\mathcal{O}_{X}$-privileged polycylinder $K \subset \mathbb{C}^{n}$ we set

$$
\mathscr{X}_{K}:=\mathscr{X} \cap(S \times K), \quad \mathscr{X}_{K, T}:=\mathscr{X}_{T} \cap(T \times K)
$$

if $T$ is a complex space over $S$. Shrinking $S$ suitably around 0 , we may assume that $K$ is $\mathcal{O}_{X(s)}$-privileged for all $s \in S$. According to $\S 2, \mathscr{X}_{K}$ and $\mathscr{X}_{K, T}$ are polycylinder spaces, and we consider the morphism space

$$
M_{S}\left(\mathscr{X}_{K}, \mathscr{Y}\right)
$$

which represents the functor

$$
\mathfrak{B a n} / S \ni T \longmapsto \operatorname{Mor}_{T}\left(\mathscr{X}_{K, T}, \mathscr{Y}_{T}\right) \text {. }
$$

We shall suppose that the following condition holds:

(A 3) There is a neighbourhood basis of $\mathcal{O}_{X}$-privileged polycylinders $K$ around $0 \in \boldsymbol{C}^{n}$ and a Banach analytic subspace

$$
M_{K} \longrightarrow M_{S}\left(\mathscr{X}_{K}, \mathscr{Y}\right)
$$

for each $K$, containing $\xi_{0}$, with the following properties:

(i) (Smoothness) The canonical map

$$
T\left(M_{K} / S\right) \longrightarrow M_{K}
$$

is smooth in a neighbourhood of $\xi_{0}$.

(ii) If $\varphi:(T, 0) \longrightarrow\left(M_{K}, \xi_{0}\right)$ is an $S$-morphism and $\tilde{\varphi}: \mathscr{X}_{K, T} \longrightarrow \mathscr{Y}_{T}$ 
the associated morphism, then the restriction of $\tilde{\varphi}$ to the interior of $K$ is a structure preserving map, i。 e. is in $\mathfrak{M}_{T}(X \cap \stackrel{\circ}{K})$ 。

(iii) If $\tilde{\varphi} \in \mathfrak{M}_{T}(X \cap K)$ is a morphism defined in a neighbourhood of $X \cap K \subset \mathscr{X}_{T}$, extending $\xi_{0}$ and if

$$
\varphi:(T, 0) \longrightarrow\left(M_{S}\left(\mathscr{X}_{K}, \mathscr{Y}\right), \xi_{0}\right)
$$

is the correspoding $S$-map, then $\varphi$ factorizes over $\left(M_{K}, \xi_{0}\right)$.

We shall show

(3.1) Proposition. If $\left(\mathfrak{M}_{T}\right)_{T}$ is a datum of structure preserving maps satisfying (A3), then for each polycylinder $K$ as in (A3) there is a closed subspace $\left(S_{K}, 0\right) \longrightarrow(S, 0)$ such that $M_{K}$ maps into $S_{K}$ and

$$
M_{K} \longrightarrow S_{K}
$$

is smooth in a neighbourhood of $\xi_{0}$.

Proof. By (A3) (i) $T\left(M_{K} / S\right) \rightarrow M_{K}$ is smooth in a neighbourhood of $\xi_{0}$. Applying the subimmersivity criterion of Douady (see [Dou ${ }_{2}$ ] Théorème 1$),\left(M_{K}, \xi_{0}\right)$ is smooth over a subgerm $\left(S_{K}, 0\right)$ of $(S, 0)$.-

(3.2) Corollary。 Let $\left(\mathfrak{M}_{T}\right)_{T}$ be a datum of structure preserving maps satisfying (A3). Then for each polycylinder $K \subset \mathbb{C}^{n}$ as in (A3), the conditions (1.7)(1), (2) are fulfilled.

Proof. Property (1.7) (1) easily follows from the universal property of $M_{S}\left(\mathscr{X}_{K}, \mathscr{Y}\right)$ and (A3) (iii), (3.1). In order to verify (1.7)(2), let $\left(T^{\prime}, 0\right) \longrightarrow(T, 0)$ be an $S_{K}$-embedding and $\varphi^{\prime} \in \mathfrak{M}_{T^{\prime}}(X \cap K)$ a structure preserving map which is defined in some neighbourhood of $X \cap K \subset \mathscr{X}_{T}$, and extends $\xi_{0}$. Consider the following commutative diagram

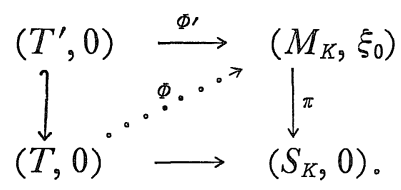

Here $\Phi^{\prime}$ is the map associated to $\varphi^{\prime}$. By the smoothness of $\pi$ there exists a lifting $\Phi$ of $\Phi^{\prime}$ as indicated by the dotted arrow. Obviously (A3) (ii) gives property (1.7)(2).- 
Putting things together we therefore obtain from (1.8):

(3.3) Theorem. If $\left(\mathscr{X}_{T}\right)_{T}$ is a datum of structure preserving maps satisfying (A3), then the functor $F$ in (1.6) is representable, i.e. there exists a maximal extension locus for $\xi_{0}$.

\section{§4. Smoothness for Deformations of Holomorphic Mappings}

As in $(1.5)$, let $(\mathscr{Z}, 0) \rightarrow(S, 0)$ be a fixed morphism of germs of complex spaces and consider morphisms

$$
\begin{aligned}
& \left(\mathscr{X}_{0}, 0\right) \stackrel{f}{\longrightarrow}\left(\mathscr{X}_{1}, 0\right) \longrightarrow(\mathscr{Z}, 0), \\
& \left(\mathscr{Y}_{0}, 0\right) \underset{g}{\longrightarrow}\left(\mathscr{Y}_{1}, 0\right) \longrightarrow(\mathscr{Z}, 0) .
\end{aligned}
$$

We assume that there is a commutative diagram

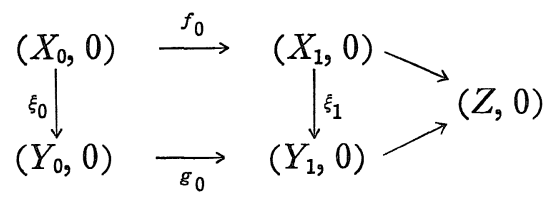

of the special fibres over $0 \in S$ and consider the datum of structure preserving maps

$$
\mathfrak{M}_{T} \subset \mathfrak{M O r}_{T}\left(\mathscr{X}_{T}, \mathscr{Y}_{T}\right)
$$

as defined in (1.5). In this section we shall always assume that the following conditions are satisfied

(4.1) (i) $\mathscr{X}_{0}, \mathscr{X}_{1}$ are $S$-flat,

(ii) the sheaf $\mathscr{E}_{x} t_{X_{0}}^{1}\left(L \xi_{0}^{*}\left(L_{\mathscr{Y}_{0} / \mathscr{Y}_{1}} \stackrel{\otimes}{=} \mathscr{O}_{\mathscr{Y}_{0}} \mathcal{O}_{Y_{0}}\right), \mathcal{O}_{X_{0}}\right)$

is finite over $X_{1}$

(iii) the canonical map

$$
\mathscr{T}^{0}\left(g / \mathscr{Z}_{;} \mathcal{O}_{\mathscr{Y}_{0}}, \mathcal{O}_{\mathscr{Y}_{1}}\right)_{(0,0)} \otimes_{\mathscr{O}_{\mathscr{Y}_{1}, 0}} \mathcal{O}_{X_{1}, 0} \longrightarrow \mathscr{T}^{0}\left(g_{0} / Z ; \mathcal{O}_{X_{0}}, \mathcal{O}_{X_{1}}\right)_{(0,0)}
$$

is surjective.

Here, by abuse of notation, $\mathscr{T}^{0}\left(g_{0} / Z ; \mathcal{O}_{X_{0}}, \mathcal{O}_{X_{1}}\right)_{(0,0)}$ stands for

$$
\mathscr{T}^{0}\left(g_{0} / Z ; \xi_{0 *}\left(\mathcal{O}_{X_{0}}\right), \xi_{1 *}\left(\mathcal{O}_{X_{1}}\right)\right)_{(0,0)} \text {. }
$$

We remark that (4.1) (ii) implies in particular that the cokernel of 
the canonical map

$$
\mathscr{H}_{6 m_{X_{0}}}\left(\xi_{0}^{*}\left(\Omega_{Y_{0} / Z}^{1}\right), \mathcal{O}_{X_{0}}\right) \longrightarrow \mathscr{H}_{0 m_{X_{0}}}\left(f_{0}^{*} \xi_{1}^{*}\left(\Omega_{Y_{1} / Z}^{1}\right), \mathcal{O}_{X_{0}}\right)
$$

is finite over $X_{1}$ (compare the second exact sequence of the proof of (1.5))。

\section{(4.2) We fix $S$-embeddings}

$$
\mathscr{X}_{i} \longrightarrow S \times \mathbb{C}^{n_{i}^{\prime}}, \quad i=0,1
$$

and we embed $\mathscr{X}_{0}$ with the product map

$$
\mathscr{X}_{0} \longrightarrow S \times \mathbb{C}^{n_{0}}, \quad n_{0}:=n_{0}^{\prime}+n_{1}^{\prime} .
$$

Moreover we put $n_{1}:=n_{1}^{\prime}, n:=n_{0}+n_{1}$ and regard $\mathscr{X}:=\mathscr{X}_{0} \times_{s} \mathscr{X}_{1}$ always as a subspace of $S \times \mathbb{C}^{n}$ via the product embedding. In particular, we get embeddings of the special fibres

$$
X_{1} \longrightarrow \mathbb{C}^{n_{1}}, \quad X_{0} \longrightarrow \mathbb{C}^{n_{0}}, \quad X \longrightarrow \mathbb{C}^{n} .
$$

For privileged chosen polycylinders $K_{i}^{\prime} \subset \mathbb{C}^{n_{i}^{\prime}}$ and after shrinking $S$, the spaces

$$
\begin{array}{ll}
\mathscr{X}_{0 K}:=\mathscr{X}_{0} \cap\left(S \times K_{0}\right), & K_{0}:=K_{0}^{\prime} \times K_{1}^{\prime} \subset \mathbb{C}^{n_{0}} \\
\mathscr{X}_{1 K}:=\mathscr{X}_{1} \cap\left(S \times K_{1}\right), & K_{1}:=K_{1}^{\prime} \subset \mathbb{C}^{n_{1}} \\
\mathscr{X}_{K}:=\mathscr{X} \cap(S \times K), & K:=K_{0} \times K_{1} \subset \mathbb{C}^{n}
\end{array}
$$

are polycylinder spaces over $S$ as considered in $\S 2$. For any space $T \rightarrow S$ over $S$, we denote by $\mathscr{X}_{K, T}$ or $T \times_{S} \mathscr{X}_{K}$ the polycylinder space $\mathscr{X}_{T} \cap(T \times K)$ over $T$, similarly for the $\mathscr{X}_{i}$. The aim of this section is to prove the following assertion.

(4.3) Theorem. If (4.1) (i)-(iii) are satisfied, then the datum $\left(\mathfrak{M}_{T}\right)_{T}$, defined in (1.5), fulfills (A3).

First we prove

(4.4) Lemma. Assume that (4.1) (i) holds. Then, for suitably chosen polycylinders $K_{\imath}^{\prime}$, there are Banachanalytic subspaces $M=M_{K}$ of $M_{S}\left(\mathscr{X}_{K}, \mathscr{Y}\right)$ such that conditions (A3) (ii), (iii) are true.

Proof. We shall construct $M$ as a subspace of the product 


$$
M_{0} \times{ }_{S} M_{1}, \quad M_{i}:=M_{S}^{\mathscr{P}}\left(\mathscr{X}_{i K}, \mathscr{Y}_{i}\right),
$$

which is contained in $M_{S}\left(\mathscr{X}_{K}, \mathscr{Y}\right)$.

The composition with $f$ resp. $g$ gives $S$-morphisms

$$
\begin{array}{ll}
\Gamma_{g}: M_{0} \longrightarrow M_{S}^{\mathscr{Q}}\left(\mathscr{X}_{0 K}, \mathscr{Y}_{1}\right), & h \mapsto g \circ h \\
{ }_{f} \Gamma: M_{1} \longrightarrow M_{S}^{\mathscr{P}}\left(\mathscr{X}_{0 K}, \mathscr{Y}_{1}\right), & h \mapsto h \circ f
\end{array}
$$

and we define $M=M_{K}$ to be the kernel of the double arrow

$$
M_{0} \times_{S} M_{1} \underset{f^{\Gamma}}{\stackrel{\Gamma_{g}}{\longrightarrow}} M_{S}^{\mathscr{F}}\left(\mathscr{X}_{0 K}, \mathscr{Y}_{1}\right)
$$

Set-theoretically, $M$ consists of all triples $\left(s, \zeta_{0}, \zeta_{1}\right)$ where $s \in S$ is a point and

$$
\zeta=\left(\zeta_{0} \times \zeta_{1}\right): \mathscr{X}_{0 K}(s) \times \mathscr{X}_{1 K}(s) \longrightarrow \mathscr{Y}_{0}(s) \times \mathscr{Y}_{1}(s)
$$

is a $\mathscr{Z}(s)$-morphism which maps the graph of $f(s)$ to the graph of $g(s)$, i. e.

$$
g(s) \circ \zeta_{0}=\zeta_{1} \circ f(s) .
$$

In the following, the point $\left(0, \xi_{0}, \xi_{1}\right)$ in $M$ will always be denoted by $\xi$. The pullback of the universal morphism

$$
M_{i} \times_{s} \mathscr{X}_{i K} \longrightarrow \mathscr{Y}_{i}, \quad i=0,1
$$

gives us a $\mathscr{Z}$-morphism

$$
\Phi_{i}: M \times{ }_{s} \mathscr{X}_{i K} \longrightarrow \mathscr{Y}_{1}, \quad i=0,1,
$$

such that $g_{\circ} \Phi_{0}=\Phi_{1} \circ\left(\operatorname{id}_{M} \times_{S} f_{K}\right)$, and $M, \Phi_{0}, \Phi_{1}$ have the following universal property: For every Banachanalytic space $T$ over $S$ and a commutative diagram

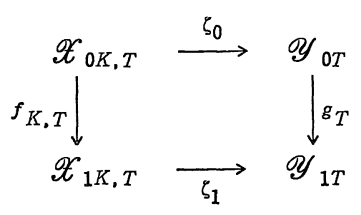

of $\mathscr{Z}_{T}$-morphisms, there is a unique $S$-map $h: T \rightarrow M$ such that $h^{*}\left(\Phi_{i}\right)=\zeta_{i}, i=0,1$. This immediately implies the conditions (A3) (ii), (iii).-

For the verification of the most difficult part (A3) (i) we need some preparations. 
(4. D) Definition. Let $\mathscr{E}_{i}, i=0,1$, be a coherent sheaf on $\mathscr{Y}_{i}$, admitting a global finite presentation. Then, according to $\S 2$, we define

$$
\mathbb{V}_{i}\left(M ; \mathscr{E}_{i}\right):=M \times_{M_{i}} V\left(M_{i} ; \mathscr{E}_{i}\right), \quad i=0,1
$$

and call $\mathbb{V}_{i}\left(M ; \mathscr{E}_{i}\right)$ the Banach fibre space over $M$ associated to $\mathscr{E}_{i \circ}$ We note that for a good choice of polycylinders, these Banach fibre spaces are direct in $\xi$ with fibre

$$
\mathbb{V}_{i}\left(M ; \mathscr{E}_{i}\right)(\xi) \cong B\left(K_{i}, \mathscr{H}_{o m_{X_{i}}}\left(\xi_{i}^{*}\left(\mathscr{E}_{i}(0)\right), \mathcal{O}_{X_{i}}\right)\right) .
$$

(4.6) Remark. Put $\mathscr{F}:=\mathscr{E}_{1}$. Then there is a canonical morphism

$$
\gamma_{K}: \mathbb{V}_{1}(M ; \mathscr{F}) \longrightarrow V_{0}\left(M ; g^{*}(\mathscr{F})\right)
$$

of Banach fibre spaces over $M$.

Proof. We fix a finite presentation

$$
\mathcal{O}_{\mathscr{V}_{1}}^{q} \stackrel{\left(h_{i j}\right)}{\longrightarrow} \mathcal{O}_{\mathscr{Y}_{1}}^{p} \longrightarrow \mathscr{F} \longrightarrow 0
$$

with a $(q \times p)$-matrix $\left(h_{i j}\right)$. Putting

$$
h^{(\nu)}:=h \circ \Phi_{\nu}, \quad \nu=0,1, \quad h \in \Gamma\left(\mathscr{Y}_{\nu}, \mathcal{O}_{\mathscr{Y}_{\nu}}\right)
$$

it is sufficient to verify that the diagram

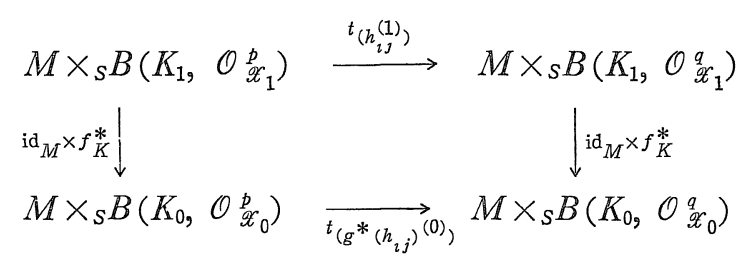

commutes. But this follows at once from the formula

$$
g \circ \Phi_{0}=\Phi_{1} \circ\left(\operatorname{id}_{M} \times_{S} f_{K}\right)_{\circ}-
$$

(4.7) Definition。 Let $\mathscr{E}_{2}$ be as in (4.5) and let $\varphi: g^{*}\left(\mathscr{E}_{1}\right) \rightarrow \mathscr{E}_{0}$ be a homomorphism. Then we denote by

$$
V\left(M ; \mathscr{E}_{0}, \mathscr{E}_{1}\right)
$$

the Banach fibre space over $M$ which is the kernel of the map

$$
\varphi_{K}-\gamma_{K}: \mathbb{V}_{0}\left(M ; \mathscr{E}_{0}\right) \times_{M} \mathbb{V}_{1}\left(M ; \mathscr{E}_{1}\right) \longrightarrow \mathbb{V}_{0}\left(M ; g^{*}\left(\mathscr{E}_{1}\right)\right) \text { 。 }
$$

Here $\varphi_{K}$ is the morphism induced by $\varphi$ (see $\S 2$ ). 
This construction is important because of

(4.8) Lemma. The relative tangent space $T(M / S)$ is canonically isomorphic to

$$
V\left(M ; \Omega_{\mathscr{O}_{0} / \mathscr{Z}}^{1}, \Omega_{\mathscr{O}_{1} / \mathscr{Z}}^{1}\right)
$$

Proof. From (2.3) we obtain a canonical isomorphism

$$
M \times_{M_{i}} T\left(M_{i} / S\right) \cong V_{i}\left(M ; \Omega_{\mathscr{Y}}^{1} / \mathscr{E}\right) .
$$

If we set $M_{2}:=M_{S}^{\mathscr{P}}\left(\mathscr{X}_{0 K}, \mathscr{Y}_{1}\right)$, then there is an isomorphism

$$
M \times_{M_{2}} T\left(M_{2} / S\right) \cong V_{0}\left(M ; g^{*}\left(\Omega_{\mathscr{W}_{1} / \mathscr{E}}^{1}\right)\right),
$$

which can be seen as follows: It $\Omega_{\mathscr{W}_{1} / \mathscr{E}}^{1}$ has a presentation

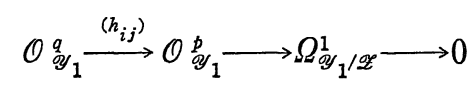

then $M \times_{M_{2}} T\left(M_{2} / S\right)$ is the kernel of the morphism

$$
M \times{ }_{S} B\left(K_{0}, \mathcal{O}_{\mathscr{X}_{0}}^{p}\right) \stackrel{\left(\bar{h}_{i j}\right)}{\longrightarrow} M \times{ }_{s} B\left(K_{0}, \mathcal{O}_{\mathscr{X}_{0}}^{q}\right)
$$

where

$$
\tilde{h}_{i j}=h_{j i} \circ g^{\circ} \Phi_{0}=\left(g^{*}\left(h_{j i}\right)\right)^{(0)} .
$$

Now, since $M$ is the kernel of the double arrow $M_{0} \times{ }_{s} M_{1} \underset{{ }_{f^{\Gamma}}}{\stackrel{\Gamma_{g}}{\longrightarrow}} M_{2}$, the tangent space $T(M / S)$ is the kernel of the map

$$
T\left(M_{0} / S\right) \times_{M_{0}} M \times_{M_{1}} T\left(M_{1} / S\right)_{\overrightarrow{T T^{-T} f} \underset{T}{\longrightarrow}}^{\longrightarrow} M \times_{M_{2}} T\left(M_{2} / S\right) .
$$

But under the isomorphisms above this map can be identified with $\varphi_{K}-\gamma_{K}$ according to (2.4) and (2.5).-

For the verification of condition (A3) (i) we shall prove

(4.9) Proposition. Let $\mathscr{E}_{i}, i=0,1$, be a coherent sheaf on $\mathscr{Y}_{i}$, admitting a global finite resolution, and $\varphi: g^{*}\left(\mathscr{E}_{1}\right) \rightarrow \mathscr{E}_{0}$ a homomorphism such that the following conditions hold with $\overline{\mathscr{E}}_{i}:=\xi_{i}^{*}\left(\mathscr{E}_{i}(0)\right)$

(i) The cokernel $\overline{\mathscr{C}}$ of the map

$$
\bar{\psi}:=\bar{\varphi}^{\vee}: \mathscr{H}_{\operatorname{Com}_{X_{0}}}\left(\overline{\mathscr{E}}_{0}, \mathcal{O}_{X_{0}}\right) \longrightarrow \mathscr{H}_{m_{X_{0}}}\left(f_{0}^{*}\left(\overline{\mathscr{E}}_{1}\right), \mathcal{O}_{X_{0}}\right)
$$

is finite over $X_{1}$. 
(ii) The canonical map

$$
\begin{gathered}
\mathscr{H}_{\operatorname{fom}_{\left(\mathscr{V}_{0}, \mathscr{U}_{1}\right)}}\left(\left(\mathscr{E}_{0}, \mathscr{E}_{1}\right),\left(\mathcal{O}_{\mathscr{Y}_{0}}, \mathcal{O}_{\mathscr{Y}_{1}}\right)\right)_{(0,0)} \otimes_{\mathscr{O}_{\mathscr{V}_{1}, 0}} \mathcal{O}_{X_{1}, 0} \\
\longrightarrow \mathscr{H}_{\left.\operatorname{Com}_{\left(X_{0}, X_{1}\right.}\right)}\left(\left(\overline{\mathscr{E}}_{0}, \overline{\mathscr{E}}_{1}\right),\left(\mathcal{O}_{X_{0},} \mathcal{O}_{X_{1}}\right)\right)_{(0,0)}
\end{gathered}
$$

is surjective.

Then, for suitably privileged polycylinders $K_{i}^{\prime}$, the Banach fibre space $\mathbb{V}\left(M ; \mathscr{E}_{0}, \mathscr{E}_{1}\right)$ is a locally trivial Banach bundle over $M$.

(4.10) Corollary. Under the assumptions of (4.1), and for suitable polycylinders $K_{i}^{\prime}$, condition (A3) (i) is satisfied for $M=M_{K}$.

Proof of (4.10). We apply (4.9) to the case $\mathscr{E}_{i}:=\Omega_{\mathscr{O}_{i} / \mathscr{L}}^{1}, i=0,1$. Then (i) and (ii) are satisfied because of (4.1) (ii), (iii). This yields the desired result.-

Let now $\mathscr{E}_{1}$ be as in (4.9) and

$$
\begin{aligned}
& E_{1}:=\mathbb{V}_{0}\left(M ; \mathscr{E}_{0}\right) \times_{M} \mathbb{V}_{1}\left(M ; \mathscr{E}_{1}\right), \\
& E_{2}:=\mathbb{V}_{0}\left(M ; g^{*}\left(\mathscr{E}_{1}\right)\right) .
\end{aligned}
$$

The proof of (4.9) immediately follows from (6.4) and

(4.11) Lemma. There is a morphism $\alpha_{K}: E_{0} \rightarrow E_{1}$ of Banach fibre spaces over $M$ with the properties

(i) $E_{0}$ is a Banach bundle over $M$,

(ii) the composition $\beta_{K} \circ \alpha_{K}$ is zero where $\beta_{K}:=\varphi_{K}-\gamma_{K}$, (see (4.7)),

(iii) the sequence of fibres over $\xi$

$$
E_{0}(\xi) \stackrel{\alpha_{K}^{(\xi)}}{\longrightarrow} E_{1}(\xi) \stackrel{\beta_{K}(\xi)}{\longrightarrow} E_{2}(\xi)
$$

is a direct exact sequence of Banach spaces.

Proof. We consider the following diagram with exact rows and columns

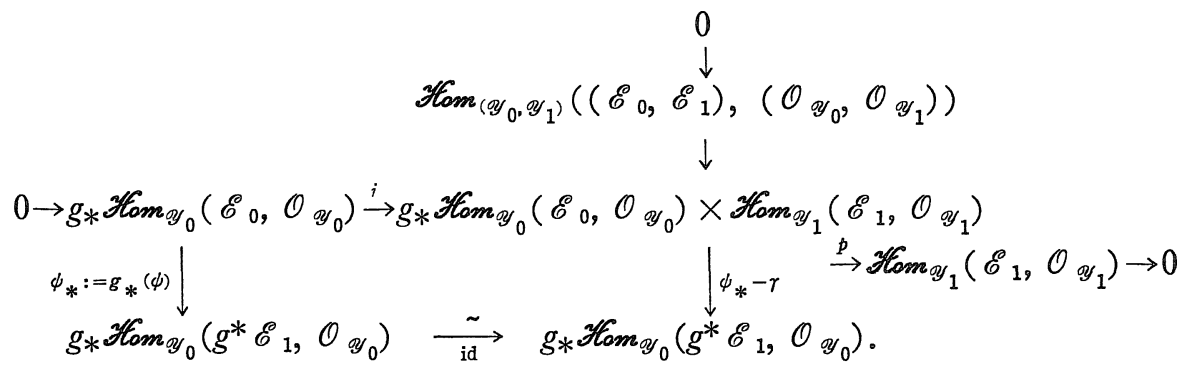


Here $i$ resp. $p$ denotes the canonical injection resp. projection, and $\phi$ is the map dual to $\varphi$. Similarly, there is also a diagram

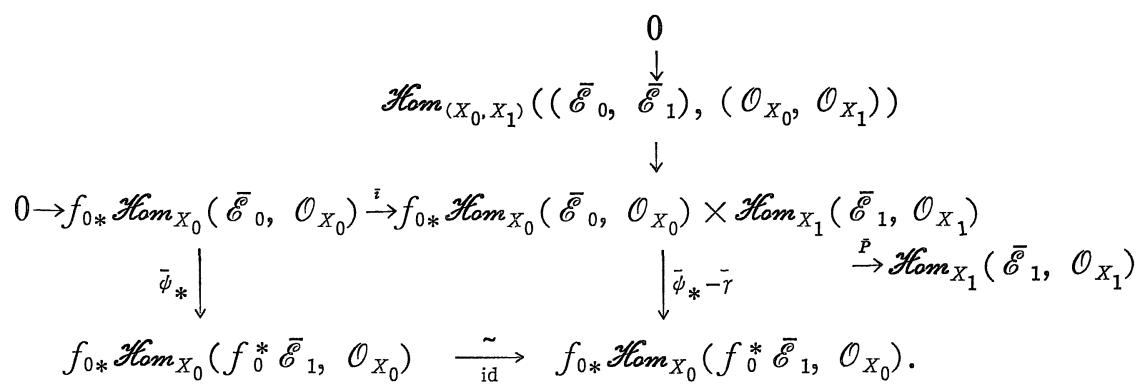

The induced map $\mathscr{H}_{\mathscr{O}_{1}}\left(\mathscr{E}_{1}, \mathcal{O}_{\mathscr{Y}_{1}}\right)_{0} \rightarrow \operatorname{Coker}\left(\phi_{*}\right)_{0}$ is a map of $\mathscr{O}_{\mathscr{O}_{1}, 0^{-}}$ modules and the kernel $\mathscr{K}$ is generated by a finite number of germs $\sigma_{1}, \ldots, \sigma_{q}$, which may be assumed to come from sections $\tau_{1}, \ldots, \tau_{q}$ in $\mathscr{H}_{\mathrm{Cm}_{\left(\mathscr{Y}_{0}, \mathscr{Y}_{1}\right)}}\left(\left(\mathscr{E}_{0}, \mathscr{E}_{1}\right),\left(\mathcal{O}_{\mathscr{Y}_{0}}, \mathcal{O}_{\mathscr{Y}_{1}}\right)\right)$. We call the homomorphism given by $\left(\tau_{1}, \ldots, \tau_{q}\right)$

$$
\alpha_{1}: \mathcal{O}_{\mathscr{Y}_{1}}^{q} \longrightarrow \mathscr{H}_{\left.m_{\left(\mathscr{Y}_{0}, \mathscr{Y}_{1}\right.}\right)}\left(\left(\mathscr{E}_{0}, \mathscr{E}_{1}\right),\left(\mathcal{O}_{\mathscr{Y}_{0}}, \mathcal{O}_{\mathscr{Y}_{1}}\right)\right) \text {. }
$$

Moreover, there is a homomorphism

$$
\alpha_{0}: \mathcal{O}_{\mathscr{Y}_{0}}^{p} \longrightarrow \mathscr{H}_{0 m_{\mathscr{Y}_{0}}}\left(\mathscr{E}_{0}, \mathcal{O}_{\mathscr{Y}_{0}}\right)
$$

which maps surjectively onto the kernel of $\phi$. We set

$$
E_{0}:=M \times_{s} B\left(K_{0}, \mathcal{O}_{\mathscr{X}_{0}}^{p}\right) \times{ }_{s} B\left(K_{1}, \mathcal{O}_{\mathscr{X}_{1}}^{q}\right)
$$

and denote by

$$
\alpha_{K}: E_{0} \longrightarrow E_{1}
$$

the morphism induced by $\left(\alpha_{0}, \alpha_{1}\right)$. Obviously, this map factorizes over $\mathbb{V}\left(M ; \mathscr{E}_{0}, \mathscr{E}_{1}\right)$ and so (ii) is satisfied. Since (i) is fulfilled by definition, it remains to verify (iii). For this, we consider the following diagram

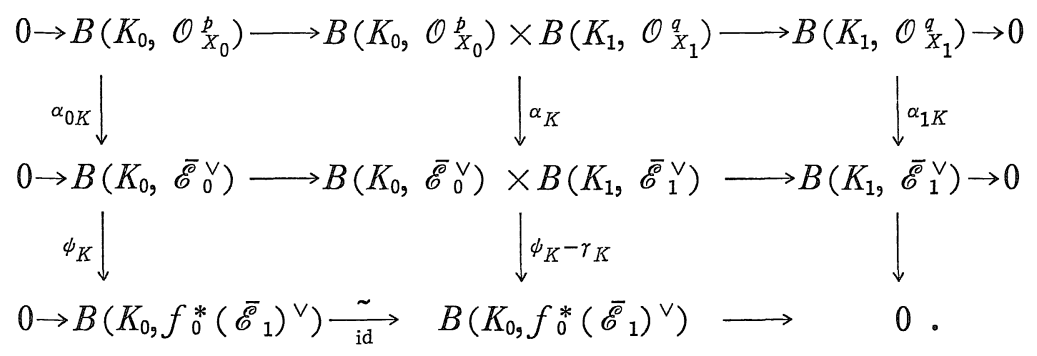


Observe that the middle column is just the fibre sequence $E_{0}(\xi) \rightarrow E_{1}(\xi)$ $\rightarrow E_{2}(\xi)$. By $(6.5)$ it suffices to prove:

(1) $\alpha_{0 K}, \alpha_{1 K}, \phi_{K}$ are direct,

(2) the induced map

$$
\operatorname{Ker}\left(\alpha_{1 K}\right) \longrightarrow \operatorname{Ker}\left(\phi_{K}\right) / \operatorname{Im}\left(\alpha_{0 K}\right)
$$

is direct and surjective,

(3) the induced map

$$
\operatorname{Coker}\left(\alpha_{1 K}\right) \longrightarrow \operatorname{Coker}\left(\phi_{K}\right)
$$

is direct and injective.

If we assume that $K_{0}, K_{1}$ are appropriate privileged, then (1) is true. For the verification of (2) and (3) we have to look at first at the following diagram of germs in the distinguished points (which are suppressed here)

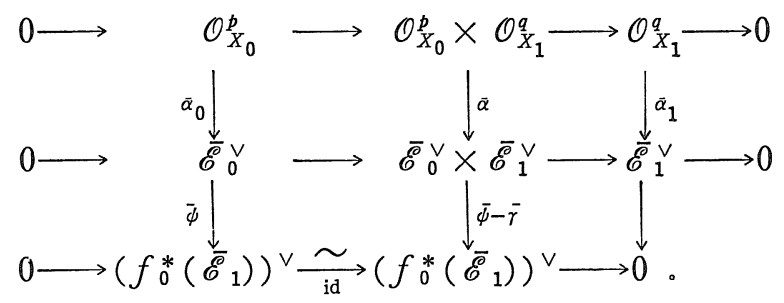

We notice that the second column is exact in the middle term. This is seen in the following way: Fix $\tilde{\omega} \in\left(\overline{\mathscr{E}}_{0}^{\vee} \times \overline{\mathscr{E}}_{1}^{\vee}\right)_{(0,0)}$ with $(\bar{\psi}-\bar{\gamma})(\tilde{\omega})=0$. Using condition (4.9) (ii) we may assume that $\tilde{\omega}$ is induced by an element $\omega$ in $\mathscr{H}_{\mathscr{C}_{\left(\mathscr{O}_{0}, \mathscr{V}_{1}\right)}}\left(\left(\mathscr{E}_{0}, \mathscr{E}_{1}\right),\left(\mathcal{O}_{\mathscr{Y}_{0}}, \mathscr{O}_{\mathscr{Y}_{1}}\right)\right)_{(0,0)}$. By the construction of $\alpha=\left(\alpha_{0}, \alpha_{1}\right)$ we get that $\omega$ is in the image of $\left(g_{*}\left(\alpha_{0}\right), \alpha_{1}\right)$. This implies $\tilde{\omega} \in \operatorname{Im}(\bar{\alpha})$. Now we obtain the exact sequences

$$
\operatorname{Ker}\left(\bar{\alpha}_{1}\right)_{0} \longrightarrow \operatorname{Ker}(\bar{\psi}) / \operatorname{Im}\left(\bar{\alpha}_{0}\right)_{0} \longrightarrow 0
$$

$0 \longrightarrow \operatorname{Coker}\left(\bar{\alpha}_{1}\right)_{0} \longrightarrow \operatorname{Coker}(\bar{\phi})_{0}$,

and so $\operatorname{Ker}(\bar{\psi}) / \operatorname{Im}\left(\bar{\alpha}_{0}\right)_{0}$ is finite over $\mathcal{O}_{x_{1}, 0}$ (also Coker $(\bar{\phi})_{0}$ by assumption). Hence we have

$$
\begin{aligned}
\operatorname{Ker}\left(\psi_{K}\right) / \operatorname{Im}\left(\alpha_{0 K}\right) & \cong B\left(K_{0}, \operatorname{Ker}(\bar{\psi}) / \operatorname{Im}\left(\bar{\alpha}_{0}\right)\right) \\
& \cong B\left(K_{1}, f_{0 *}\left(\operatorname{Ker}(\bar{\psi}) / \operatorname{Im}\left(\bar{\alpha}_{0}\right)\right)\right),
\end{aligned}
$$

and similarly

$$
\operatorname{Coker}\left(\phi_{K}\right) \cong B\left(K_{1}, f_{0 *}(\overline{\mathscr{C}})\right),
$$


see [Fl], II, (6.4). So, if $K_{1}$ is chosen privileged for the sheaf morphisms

$$
\begin{aligned}
& \operatorname{Ker}\left(\bar{\alpha}_{1}\right) \longrightarrow f_{0 *}\left(\operatorname{Ker}(\bar{\phi}) / \operatorname{Im}\left(\bar{\alpha}_{0}\right)\right), \\
& \operatorname{Coker}\left(\bar{\alpha}_{1}\right) \longrightarrow f_{0 *}(\overline{\mathscr{C}})
\end{aligned}
$$

we can achieve that (2) and (3) are satisfied. This proves (4.11).-

\section{§. Applications}

Let

$$
\begin{aligned}
& \left(\mathscr{X}_{0}, 0\right) \stackrel{f}{\longrightarrow}\left(\mathscr{X}_{1}, 0\right) \longrightarrow \\
& \left(\mathscr{Y}_{0}, 0\right) \underset{g}{\longrightarrow}\left(\mathscr{Y}_{1}, 0\right)
\end{aligned}(\mathscr{Z}, 0) \longrightarrow(S, 0)
$$

and

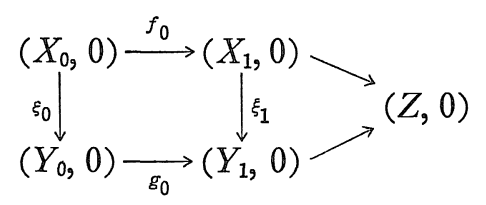

be as in (1.5). We consider the functor

$$
F: \mathfrak{U} \mathfrak{n} /(S, 0) \longrightarrow \text { gets }
$$

given by $F((T, 0)):=\{1\}$, if there exists a commutative diagram

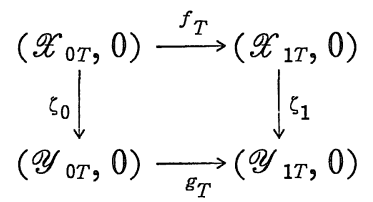

of $\mathscr{Z}_{T}$-morphisms such that $\left(\zeta_{0}, \zeta_{1}\right)$ induces $\left(\xi_{0}, \xi_{1}\right)$ in the special fibre, and $F((T, 0)):=\emptyset$ otherwise.

If $\left(\mathscr{X}_{T}\right)_{T}$ is the datum of structure preserving maps as described in (1.5), then $F$ is just the functor defined in (1.6). Using the representability theorem (3.3) and the smoothness theorem (4.3) we obtain

(5.1) Theorem. Assume that the following conditions are satisfied

(1) $\mathscr{X}_{0}, \mathscr{X}_{1}$ are S-flat, 
(2) the sheaf $\mathscr{E}_{x \in X_{X_{0}}^{1}}\left(L \xi_{0}^{*}\left(L_{\mathscr{Y}_{0} / \mathscr{Y}_{1}} \stackrel{\otimes}{=} \mathscr{O}_{\mathscr{Y}_{0}} \mathcal{O}_{Y_{0}}\right), \mathcal{O}_{X_{0}}\right)$

is finite over $X_{1}$,

(3) the canonical map

$$
\mathscr{T}^{0}\left(g / \mathscr{Z} ; \mathcal{O}_{\mathscr{Y}_{0}}, \mathcal{O}_{\mathscr{Y}_{1}}\right)_{(0,0)} \otimes_{\mathcal{O}_{\mathscr{Y}_{1,0}}} \mathcal{O}_{X_{1,0}} \longrightarrow \mathscr{T}^{0}\left(g_{0} / Z ; \mathcal{O}_{X_{0}}, \mathcal{O}_{X_{1}}\right)_{(0,0)}
$$

is surjective.

Then the functor $F$ is representable by a closed subgerm $\left(S^{\prime}, 0\right)$ of $(S, 0)$.

By applying (5.1) to the special case $f=\mathrm{id}_{\mathscr{X}_{0}}, g=\mathrm{id}_{\mathscr{Y}_{0}}$ we obtain theorem (0.1) mentioned in the introduction. We are going to show how to obtain a similar result for modules: Consider the situation described in (1.4), i, e.

$$
\xi:(\mathscr{X}, 0) \longrightarrow(\mathscr{Y}, 0)
$$

is a $(S, 0)$-morphism of germs of complex spaces with special fibres $X$ resp. $Y$ over $0 \in S$, and $\mathscr{F}$ resp. $\mathscr{G}$ are coherent $\mathcal{O}_{\mathscr{X}}$ resp. $\mathcal{O}_{\mathscr{Y}-}$ modules together with a homomorphism

$$
\varphi_{0}: \xi_{0}^{*}\left(\mathscr{G}_{0}\right) \longrightarrow \mathscr{F}_{0}
$$

of $\mathcal{O}_{X}$-modules. Here the index " 0 " denotes the restriction to the special fibre. We get from (5.1)

(5.2) Theorem. Assume that the following condition are satisfied

(1) $\mathscr{F}$ is $S$-flat,

(2) the canonical map induced by $\varphi_{0}$

$$
\mathscr{H}_{\text {om }}(\mathscr{G}, \mathscr{G})_{0} \otimes_{\mathscr{O}_{\mathscr{Y}, 0}} \mathcal{O}_{X, 0} \longrightarrow \mathscr{H}_{\mathfrak{C} m_{X}}\left(\xi_{0}^{*}\left(\mathscr{G}_{0}\right), \mathscr{F}_{0}\right)_{0}
$$

is surjective.

Then there is a subspace $\left(S^{\prime}, 0\right) \longrightarrow(S, 0)$ and a homomorphism $\varphi: \xi_{S^{\prime}}^{*}\left(\mathscr{G}_{S^{\prime}}\right) \rightarrow \mathscr{F}_{S^{\prime}}$, extending $\varphi_{0}$, with the following universal property: If $\alpha:(T, 0) \rightarrow(S, 0)$ is a morphism such that $\varphi_{0}$ admits an extension $\phi: \xi_{T}^{*}\left(\mathscr{G}_{T}\right) \rightarrow \mathscr{F}_{T}$, then $\alpha$ factorizes over $\left(S^{\prime}, 0\right)$ 。

Proof. First of all we remark that we may assume $\mathscr{X}$ to be flat over $S$. Indeed, there exists a commutative diagram of $S$-spaces

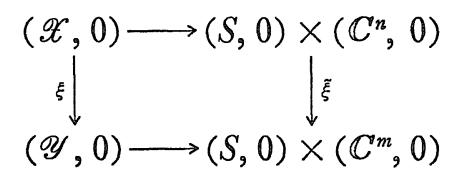


and we can replace $(\mathscr{X}, 0)$ resp. $(\mathscr{Y}, 0)$ by $(S, 0) \times\left(\boldsymbol{C}^{n}, 0\right)$ resp. $(S, 0) \times\left(\boldsymbol{C}^{m}, 0\right)$. It is sufficient to verify the conditions in (5.1) for the diagram

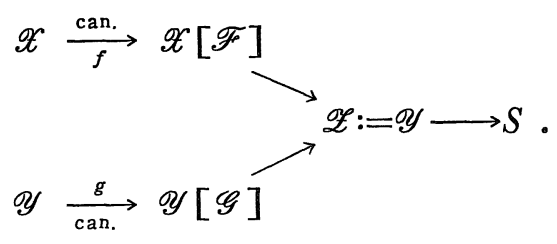

Since $f$ and $g$ are finite morphisms, (5.1) (2) is fulfilled. For the verification of (5.1) (3), we notice that one has canonical isomorphisms

$$
\begin{aligned}
& \mathscr{T}^{0}\left(g / \mathscr{Z} ; \mathcal{O}_{\mathscr{Y}}, \mathcal{O}_{\mathscr{Y}[\mathscr{G}]}\right)_{(0,0)} \cong \mathscr{H}_{\mathscr{m} m_{\mathscr{Y}}}(\mathscr{G}, \mathscr{G})_{0}, \\
& \mathscr{T}^{0}\left(g_{0} / Z ; \mathcal{O}_{X}, \mathcal{O}_{X\left[\mathscr{F}_{0}\right]}\right)_{(0,0)} \cong \mathscr{H}_{m_{X}}\left(\xi_{0}^{*}\left(\mathscr{G}_{0}\right), \mathscr{F}_{0}\right)_{0},
\end{aligned}
$$

as the reader may easily check. So (5.1)(3) follows from our assumption (2).-

The most important applications of the results above are to locally trivial deformations. First we consider deformations of holomorphic mappings.

(5.3) Theorem. Let

$$
\left(\mathscr{X}_{0}, 0\right) \stackrel{f}{\longrightarrow}\left(\mathscr{X}_{1}, 0\right) \longrightarrow(S \times Z, 0)
$$

be a deformation of the map germ $f_{0} / Z$ over $(S, 0)$ such that $T^{1}\left(X_{0} / X_{1}\right.$, $\left.\mathcal{O}_{X_{0}}\right)_{0}$ is a finite $\mathcal{O}_{X_{1}, 0}$-module. Then there is a maximal subspace $\left(S^{\prime}, 0\right) \longrightarrow(S, 0)$ such that $f_{S^{\prime}} / S^{\prime} \times Z$ is the product deformation $\operatorname{id}_{S^{\prime}} \times f_{0}$, $i$. e. there exists a commutative diagram

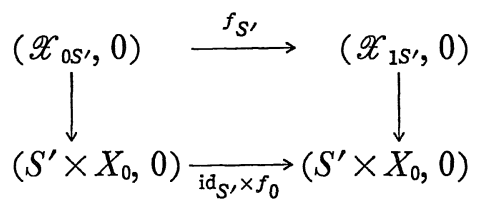

of $S^{\prime} \times Z$-morphisms, and $S^{\prime}$ is universal with this property, i. e. if $\alpha$ : $(T, 0) \rightarrow(S, 0)$ is a morphism with $f_{T} \cong \mathrm{id}_{T} \times f_{0}$ then $\alpha$ factorizes over $\left(S^{\prime}, 0\right)$.

Proof. We apply (5.1) to the case

$$
\mathscr{Y}_{0}:=S \times X_{0}, \quad \mathscr{Y}_{1}:=S \times X_{1}, \quad \mathscr{Z}:=S \times Z, \quad g:=\mathrm{id}_{s} \times f_{0} .
$$


The finiteness assumption implies that (5.1)(2) is satisfied. Observe that $L_{\mathscr{\mathscr { O }}_{0} / \mathscr{O}_{1}}=\mathcal{O}_{S} \hat{\otimes}_{c} L_{X_{0} / X_{1}}$. Apparently, also (5.1)(3) is fulfilled and (5.3) follows.-

(5.4) Remark. (1) By applying (5.3) to the special case $f=\mathrm{id}$, $\mathscr{X}_{0}=\mathscr{X}_{1}$, we obtain the analogous result for deformations of spaces, see $(0.2)$.

(2) The same conclusion also holds for deformations of modules: If $\mathscr{F}_{0}$ is a coherent module on $(X, 0)$ and $\mathscr{F}$ is a deformation of $\mathscr{F}_{0}$ over $(S, 0)$, i. e. $\mathscr{F}$ is a $S$-flat coherent module on $(S \times X, 0)$, then there is a maximal subspace $\left(S^{\prime}, 0\right) \subset(S, 0)$ such that

$$
\mathscr{F} \mid S^{\prime} \times X \cong q^{*}\left(\mathscr{F}_{0}\right)
$$

where $q: S^{\prime} \times X \rightarrow X$ is the canonical projection, and $S^{\prime}$ is universal with this property. The proof is established just by applying (5.3) to the situation

$$
(S \times X, 0) \underset{=\text { can. }}{\stackrel{f}{\longrightarrow}}((S \times X)[\mathscr{F}], 0) \longrightarrow(S \times X, 0) .
$$

In particular (5.3) can be used to construct locally trivial versal deformations. For the case of deformations of compact complex spaces this was already done in (0.3). A similar result holds for deformations of modules.

(5.5) Theorem. Let $\mathscr{F}_{0}$ be a coherent module with compact support on the complex space $X$. Then there exists a locally trivial semiuniversal deformation of $\mathscr{F}_{0}$.

Proof. By the theorem of Siu-Trautmann [S-T], see also [B-K] (V 4.11), there exists a semiuniversal deformation $\mathscr{F}^{*}$ of $\mathscr{F}_{0}$ over some base space $\left(S^{*}, 0\right)$. Using the same method as in the proof of (0.3) and in addition (5.4)(2) we get a universal subgerm $(S, 0)$ $\longrightarrow\left(S^{*}, 0\right)$ such that $\mathscr{F}:=\mathscr{F} * \mid S^{\prime} \times X$ is locally trivial in all points of $\{0\} \times X$. This $\mathscr{F}$ has the desired property.-

We remark that an analogous result also holds for other situations where semiuniversal deformations exist, so e.g. for

-deformations of holomorphic mappings, cf. [Fl] (8.5), -deformations of 1-convex spaces, cf。 [B-K $]$ (V 5.2). 
We leave the straightforward formulations and verifications to the reader.

We will give two further applications of the existence of the maximal extension locus. The first one was shown by Pourcin and is an important tool for the construction of the relative Douady space in $\left[\mathrm{Pou}_{2}\right]$.

(5.6) Theorem. Let $(\mathscr{X}, 0) \longrightarrow(S, 0)$ be a map of complex space germs and $\mathscr{F} \stackrel{\varphi}{\longrightarrow} \mathscr{G}$ be a map of coherent $\mathcal{O}_{\mathscr{X}}$-modules such that $\mathscr{G}$ is $S$-flat and $\varphi / \mathfrak{m}_{s, 0} \varphi$ is the zero map. Then the functor

$$
\begin{aligned}
& F: \mathfrak{U} \mathfrak{n} /(S, 0) \longrightarrow \text { betg } \\
& F(T, 0)=\left\{\begin{array}{l}
\{1\} \text { if } \varphi_{T}=0 \\
\emptyset \text { otherwise }
\end{array}\right.
\end{aligned}
$$

is representable by a closed subspace $\left(S^{\prime}, 0\right) \subseteq(S, 0)$.

Proof. Without restriction of generality we may assume that $\mathscr{X}$ is $S$-flat. Let the index " 0 " denote restriction to the special fibre $X$ and consider the extension problem for the map $f_{0}$ of $\mathscr{X}[\mathscr{F}]$-spaces

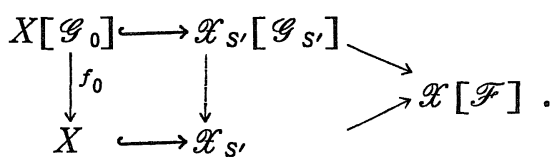

Here $f_{0}$ is the morphism given by the injection $\mathcal{O}_{X} \rightarrow \mathcal{O}_{X} \times \mathscr{G}_{0}$ and, similarly, $\mathscr{X} \rightarrow \mathscr{X}[\mathscr{F}]$ resp. $\mathscr{X}[\mathscr{G}] \rightarrow \mathscr{X}[\mathscr{F}]$ are defined by the projection $\mathcal{O}_{\mathscr{X}} \times \mathscr{F} \rightarrow \mathcal{O}_{\mathscr{X}}$ resp. $1 \times \varphi: \mathcal{O}_{\mathscr{X}} \times \mathscr{F} \rightarrow \mathcal{O}_{\mathscr{X}} \times \mathscr{G}$. By $(5.1)$ there exists the maximal extension locus $S^{\prime} \subseteq S$ for $f_{0}$. Observe that the conditions of (5.1) are trivially satisfied. Obviously $S^{\prime}$ represents the functor $F$ above.-

(5. 7) Theorem. ([Fr] $)_{\text {Let }}(\mathscr{X}, 0) \rightarrow(S, 0)$ be a morphism of complex space germs and $\mathscr{F}$ a coherent $\mathcal{O}_{\mathscr{X}}$-module. Then there is a subspace $\left(S^{\prime}, 0\right)$ in $(S, 0)$ with the following properties.

(1) $\mathscr{F}_{S^{\prime}}$ is $S^{\prime}$-flat.

(2) If $(T, 0)$ is a space germ over $(S, 0)$ and $\mathscr{F}_{T}$ is $T$-flat then $(T, 0) \rightarrow(S, 0)$ factors through $S^{\prime}$. 
Proof. Again we may assume that $\mathscr{X}$ is $S$-flat. We consider a presentation

$$
\mathcal{O}_{\stackrel{1}{\mathscr{X}}}^{r_{1}} \stackrel{\varphi}{\longrightarrow} \mathcal{O}_{\mathscr{X}}^{r_{0}} \longrightarrow \mathscr{F} \longrightarrow 0
$$

and a map $\psi_{0}: \mathcal{O}_{X}^{r_{2}} \longrightarrow \mathcal{O}_{X}^{r_{1}}$ such that

$$
\mathcal{O}_{X}^{r_{2}} \stackrel{\varphi_{0}}{\longrightarrow} \mathcal{O}_{X}^{r_{1}} \stackrel{\varphi_{0}}{\longrightarrow} \mathcal{O}_{X}^{r_{0}} \longrightarrow \mathscr{F}_{0} \longrightarrow 0
$$

is exact. By standard commutative algebra, $\mathscr{F}_{T}$ is $T$-flat iff $\phi_{0}$ can be lifted to a map

$$
\psi: \mathcal{O}_{\mathscr{X}_{T}}^{r_{2}} \longrightarrow \mathcal{O}_{\mathscr{X}_{T}}^{r_{1}}
$$

which maps into the kernel of $\varphi_{T}$. Let $\mathscr{N}$ denote the cokernel of the transposed map ${ }^{t} \varphi$. Then $\phi_{0}$ induces a map

$$
{ }^{t} \bar{\psi}_{0}=\left(\rho_{1}, \ldots, \rho_{r_{2}}\right): \mathscr{N}_{0} \longrightarrow \mathcal{O}_{X}^{r_{2}}
$$

which can also be considered as a map

$$
\mathscr{N}_{0}^{r_{2}} \stackrel{\rho}{\longrightarrow} \mathcal{O}_{X},\left(n_{j}\right) \longmapsto \sum_{j} \rho_{j}\left(n_{j}\right) \text {. }
$$

By the remark above, for a space $(T, 0) \in \mathfrak{U} \mathfrak{H} /(S, 0), \rho$ can be extended to a map

$$
\mathscr{N}_{T}^{r_{2}} \longrightarrow \mathcal{O}_{\mathscr{X}_{T}}
$$

iff $\mathscr{F}_{T}$ is $T$-flat. Hence we must show that the maximal extension locus of $\rho$ exists. For this we will verify the conditions in (5.2), i. e.

$$
\operatorname{Hom}(1, \rho): \mathscr{E}_{n d}\left(\mathscr{N}^{r_{2}}\right)_{0} \longrightarrow \mathscr{H}_{\operatorname{mon}_{X}}\left(\mathcal{N}_{0}^{r_{2}}, \mathcal{O}_{X}\right)_{0}
$$

must be surjective. Let

$$
g=\left(g_{1}, \ldots, g_{r_{2}}\right): \mathscr{N}_{0}^{r_{2}} \longrightarrow \mathcal{O}_{X}
$$

be given. By construction, $\rho_{1}, \ldots, \rho_{r_{2}}$ are generators of $\mathscr{H}_{0 m m_{X}}\left(\mathscr{N}_{0}, \mathcal{O}_{X}\right)_{0}$ and so there are $a_{i j} \in \mathcal{O}_{\mathscr{X}, 0}$ with

$$
g_{j}=\sum_{i} \tilde{a}_{i j} \circ \rho_{i 9}
$$

if $\bar{a}_{i j}$ is the residue class of $a_{i j}$ in $\mathcal{O}_{X, 0}$. Obviously, the endomorphism of $\mathscr{N}^{r_{2}}$ given by the matrix $\left(a_{i j}\right)$ then maps onto $g_{0}-$

(5.8) Remark. In the situation of (3.3), let $\left(S^{\prime}, 0\right) \subseteq(S, 0)$ be the maximal extension locus of $\xi_{0}$ and $\xi: \mathscr{X}_{S^{\prime}} \rightarrow \mathscr{Y}_{S^{\prime}}$ be a structure preserving map extending $\xi_{0}$. Then the openess of versality principle 
holds, i. e. if $s \in S^{\prime}$ is sufficiently near 0 then $\left(S^{\prime}, s\right)$ is the maximal extension locus for $\xi(s)$. This follows easily from the proof of (3.3). From this we get that this openess principle also holds in $(0.1)-(0.3)$ and (5.1)-(5.7).-

\section{§6. Appendix: Banach Fibre Spaces over Banach analytic Spaces}

(6.1) Definition. Let $\mathscr{E}$ be a Banach analytic space over the Banach analytic space $S$ and suppose that $\mathscr{E}$ is equipped with an addition, scalar multiplication and zero section

$$
\begin{gathered}
\mathscr{E} \times s \mathscr{E} \stackrel{\text { add. }}{\longrightarrow} \mathscr{E}, \\
C \times \mathscr{E} \stackrel{\text { mult. }}{\longrightarrow} \mathscr{E}, \\
S \quad \longrightarrow \mathscr{E},
\end{gathered}
$$

which satisfy the usual axioms for vector spaces relative $S$. Then $\mathscr{E}$ is called a Banach fibre space over $S$ if in addition $\mathscr{E}$ is locally in $S$ isomorphic to the kernel of a linear $S$-morphism

$$
\varphi: E \times S \longrightarrow F \times S
$$

of trivial Banach bundles over $S$.

Observe that the fibres of $\mathscr{E} \rightarrow S$ are in general not Banach spaces. E.g., if $S$ is a point and $f: E \rightarrow F$ is a linear map of Banach spaces then $\operatorname{Ker}(f)$ is a Banach fibre space, and $\operatorname{Ker}(f)$ is a Banach space in the usual sense iff $f$ is direct.

In the following, a Banach fibre space which is locally in $S$ isomorphic to a product $E \times S$ with a Banach space $E$, will always be called a (locally trivial) Banach bundle. Morphisms of Banach fibre spaces and bundles will always be considered to be linear.

Now let $\mathscr{E} \rightarrow S$ be a Banach fibre space and $s_{0} \in S$. We shall say that $\mathscr{E}$ is direct in $s_{0}$ if the fibre $\mathscr{E}\left(s_{0}\right)$ is a Banach space. If $\mathscr{E}$ is given as the kernel of a linear map $\varphi$ as above, then $\mathscr{E}$ is direct in $s_{0}$ iff $\varphi\left(s_{0}\right)$ is a direct morphism, and this is equivalent to the smoothness of the fibre $\mathscr{E}\left(s_{0}\right)$.

(6.2) Lemma. Let $\mathscr{E}$ be a Banach fibre space which is direct in 
$s_{0} \in S$ and put $E:=\mathscr{E}\left(s_{0}\right)$. Then, after shrinking $S$ as a neighborhood of $s_{0}$, there is a morphism

$$
f: E \times S \longrightarrow F \times S
$$

of trivial Banach bundles such that $\mathscr{E} \cong \mathrm{Ker}(f)$.

Proof. Suppose that $\mathscr{E}=\operatorname{Ker}(g)$, where

$$
g: E^{\prime} \times S \longrightarrow F^{\prime} \times S
$$

is a morphism of trivial Banach bundles. By our assumption there is a decomposition

$$
E^{\prime}=E \times B, \quad F^{\prime}=F \times B
$$

of Banach spaces such that for $g=\left(g_{1}, g_{2}, \mathrm{id}_{s}\right)$ one has $g_{1}\left(e, b, s_{0}\right)=0$, $g_{2}\left(e, b, s_{0}\right)=b$. Applying a bundle automorphism of $E^{\prime} \times S$, we may assume that $g_{2}(e, b, s) \equiv b$. Now with $f(e, s):=g_{1}(e, 0, s) \operatorname{Ker}(g) \cong$ $\operatorname{Ker}(f)$ and the lemma is proven.

(6. 3) Extension lemma. Let $\mathscr{E}=\operatorname{Ker}(E \times S \stackrel{f}{\longrightarrow} F \times S)$ be a Banach fibre space over $S$ and $\varphi: \mathscr{E} \longrightarrow G \times S$ a morphism of Banach fibre spaces where $G$ is a Banach space. Then, locally in $S$, there is a morphism

$$
\widetilde{\varphi}: E \times S \longrightarrow G \times S
$$

of Banach bundles over $S$, extending $\varphi$.

Proof. Since $G$ is smooth there is a $S$-morphism

$$
h: U \longrightarrow G \times S \text {, }
$$

defined in a neighborhood of $\left(0, s_{0}\right) \in E \times S$ which extends $\varphi$. Then the relative tangent map of $h$ restricted to $S$ (via the zero section)

$$
\tilde{\varphi}:=T(h)|S: T(U / S)| S \cong E \times S \longrightarrow G \times S
$$

obviously extends $\varphi$ linearly.-

(6.4) Proposition. Let

$$
\mathscr{E}_{0} \stackrel{\varphi}{\longrightarrow} \mathscr{E}_{1} \stackrel{\psi}{\longrightarrow} \mathscr{E}_{2}
$$

be a sequence of Banach fibre spaces over $S$ with the following properties

(i) $\mathscr{E}_{0}$ is a Banach bundle over $S$,

(ii) $\mathscr{E}_{1}$ and $\mathscr{E}_{2}$ are direct in $s_{0}$, 
(iii) $\phi \varphi=0$, and $\mathscr{E}_{0}\left(s_{0}\right) \stackrel{\varphi\left(s_{0}\right)}{\longrightarrow} \mathscr{E}_{1}\left(s_{0}\right) \stackrel{\psi\left(s_{0}\right)}{\longrightarrow} \mathscr{E}_{2}\left(s_{0}\right)$

is a direct exact sequence of Banach spaces.

Then $\operatorname{Ker}(\psi)$ is a Banach bundle over $S$.

Proof. If $\mathscr{E}_{1}$ and $\mathscr{E}_{2}$ are Banach bundles then this proposition is well known, see [Dou $]$, p. 21, Prop. 3. In the general case, let $\mathscr{E}_{1}$ resp. $\mathscr{E}_{2}$ be the kernel of the Banach bundle morphism

$$
F: \mathscr{E}_{1}^{\prime} \longrightarrow \mathscr{E}_{1}^{\prime \prime} \text {, }
$$

resp.

$$
G: \mathscr{E}_{2}^{\prime} \longrightarrow \mathscr{E}_{2}^{\prime \prime} \text {; }
$$

By (6.2) we may assume that $F\left(s_{0}\right)$ and $G\left(s_{0}\right)$ are the zero map. Using (6.3), there is (locally around $s_{0} \in S$ ) a morphism $\Psi$ which makes the diagram

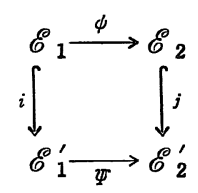

commutative. Here $i$ and $j$ are the given inclusions. We consider the following diagram

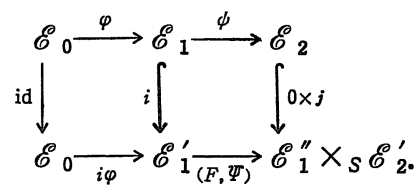

Obviously we have $\operatorname{Ker}(\psi) \cong \operatorname{Ker}(F, \Psi)$. So, by the lemma of Douady just mentioned, it suffices to show that the sequence

$$
\mathscr{E}_{0}\left(s_{0}\right) \stackrel{(i \varphi)\left(s_{0}\right)}{\longrightarrow} \mathscr{E}_{1}^{\prime}\left(s_{0}\right) \stackrel{\left(F\left(s_{0}\right), \Psi\left(s_{0}\right)\right)}{\longrightarrow} \mathscr{E}_{1}^{\prime \prime}\left(s_{0}\right) \times \mathscr{E}_{2}^{\prime}\left(s_{0}\right)
$$

is direct exact. Since $\mathscr{E}_{k}^{\prime}\left(s_{0}\right)=\mathscr{E}_{k}\left(s_{0}\right)$ for $k=1,2$, and $F\left(s_{0}\right)$ is the zero map, this follows immediately from our assumption.-

(6.5) Proposition. Let

$$
0 \longrightarrow E_{1} \longrightarrow E_{2}^{*} \longrightarrow E_{3}^{*} \longrightarrow 0
$$

be a sequence of complexes of Banach spaces with the following properties,

(i) For each $i \in \mathbb{Z}$ the sequence $0 \rightarrow E_{1}^{i} \rightarrow E_{2}^{i} \rightarrow E_{3}^{i} \rightarrow 0$ is direct exact, 
(ii) $E_{1}^{*}$ and $E_{3}^{\circ}$ are direct, i.e. all coboundary maps of these complexes are direct,

(iii) the connecting morphisms

$$
H^{i}\left(E_{3}^{*}\right) \longrightarrow H^{i+1}\left(E_{1}^{*}\right)
$$

are direct for all $i \in \mathbb{Z}$.

Then $E_{2}^{*}$ is direct too.

For the proof, see $[B-K](\mathbb{I}, 4.12)$.

\section{§7. Appendix: Extensions of Holomorphic Mappings}

In this section we shall give a brief account on the relationship between extensions of holomorphic mappings and certain Ext-groups. This correspondence-which was used several times in this paper-seems to be more or less well known, but unfortunately we could not find any precise reference even in the context of algebraic geometry. For a related situation see [IIl] vol. 1 (III 2.2). We shall state the result in a rather general way and sketch the proof only in the local case as far as it has been used in this paper.

We consider a commutative diagram of complex spaces

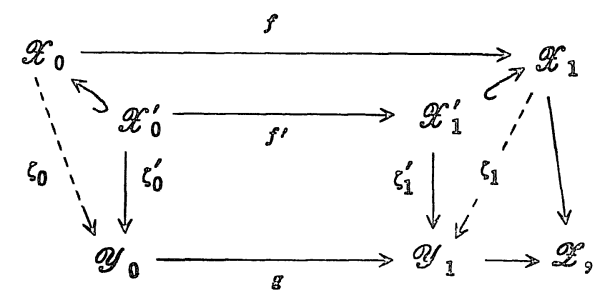

where $\mathscr{X}_{i}^{\prime} \hookrightarrow \mathscr{X}_{i}$ is an extension by the collerent $\mathcal{O}_{\mathscr{X}_{i}}^{\prime}$-module $\mathscr{M}_{i \text { 。 }}$ A pair of morphisms

$$
\zeta_{i}: \mathscr{X}_{i} \longrightarrow \mathscr{Y}_{i}, \quad i=0,1 \text {, }
$$

will be called an extension of $\left(\zeta_{0}^{\prime}, \zeta_{1}^{\prime}\right)$ if the $\zeta_{i}$ are $\mathscr{Z}$-morphisms satisfying

What we need is

$$
g \zeta_{0}=f \zeta_{1}, \quad \zeta_{i} \mid \mathscr{X}_{i}^{\prime}=\zeta_{i}^{\prime}
$$
(7. 1) Proposition.
(1) There is a natural obstruction 


$$
\text { ob }\left(\zeta_{0}^{\prime}, \zeta_{1}^{\prime}\right)
$$

for extending $\left(\zeta_{0}^{\prime}, \zeta_{1}^{\prime}\right)$ to a pair $\left(\zeta_{0}, \zeta_{1}\right)$ as above. This obstruction lies in

$$
K:=\operatorname{Ext}_{\left(\mathscr{X}_{0}^{\prime}, \mathscr{O}_{1}^{\prime}\right)}\left(\left(L \zeta_{0}^{\prime *}\left(L_{\dot{\mathscr{V}}_{0} / \mathscr{Z}}\right), L \zeta_{1}^{\prime *}\left(L_{\dot{\mathscr{V}}_{1} / \mathscr{Z}}\right)\right),\left(\mathscr{M}_{0}, \mathscr{M}_{1}\right)\right) \text {. }
$$

(2) The set of all pairs $\left(\zeta_{0}, \zeta_{1}\right)$ extending $\left(\zeta_{0}^{\prime}, \zeta_{1}^{\prime}\right)$ is a formal principal homogeneous space under the action of the group

$$
\operatorname{Ext}_{\left(\mathscr{C}_{0}^{\prime}, \mathscr{X _ { 1 }}\right)}^{0}\left(\left(L \zeta_{0}^{\prime *}\left(L_{\dot{\mathscr{O}}_{0} / \mathscr{L}}\right), L \zeta_{1}^{\prime *}\left(L_{\dot{\mathscr{Y}}_{1} / \mathscr{E}}\right)\right),\left(\mathscr{M}_{0}, \mathscr{M}_{1}\right)\right) .
$$

Proof. Since we have only used (1) we shall leave the simple proof of (2) to the reader. Moreover we shall show (1) only in the case where $\mathscr{X}_{i}$ is Stein and where there are locally closed embeddings

$$
\mathscr{Y}_{0} \longrightarrow C^{n_{0}} \times \mathscr{Z}, \quad \mathscr{Y}_{1} \longrightarrow C^{n_{1}} \times \mathscr{Z}
$$

such that $g$ is induced by the canonical projection

$$
\boldsymbol{C}^{n_{0}} \times \mathscr{Z}=\boldsymbol{C}^{n_{0}^{\prime}} \times \boldsymbol{C}^{n_{1}} \times \mathscr{Z} \stackrel{\tilde{g}}{\longrightarrow} \boldsymbol{C}^{n_{1}} \times \mathscr{Z}
$$

with $n_{0}=n_{0}^{\prime}+n_{1}$. Obviously the $\zeta_{i}^{\prime}$ can be lifted to holomorphic $\mathscr{Z}-$ maps

$$
\tilde{\zeta}_{i}: \mathscr{X}_{i} \longrightarrow C^{n_{i}} \times \mathscr{Z}
$$

such that the diagram

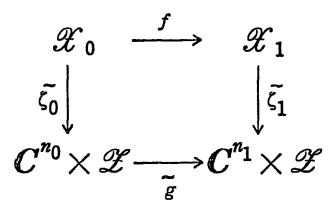

commutes. Let $\mathscr{Y}_{2}^{(1)}$ be the first infinitesimal neighborhood of $\mathscr{Y}_{i}$ in $\boldsymbol{C}^{n_{i}} \times \mathscr{Z}$ and $\mathscr{I}_{i} \subset\left(\mathscr{O}_{c^{n_{i} \times \mathscr{Z}}} \mid \mathscr{Y}_{i}\right)$ be the ideal of $\mathscr{Y}_{i}$. Since $\tilde{\zeta}_{i}\left(\mathscr{X}_{i}^{\prime}\right) \subset \mathscr{Y}_{i}$ we obtain $\tilde{\zeta}_{i}\left(\mathscr{X}_{i}\right) \subset \mathscr{Y}_{i}^{\stackrel{c}{(1)} \text { and }}$ and so $\tilde{\zeta}_{i}$ determines a pair of homomorphisms

$$
\alpha_{i}: \zeta_{i}^{\prime *}\left(\mathscr{I}_{i} / \mathscr{I}_{i}^{2}\right) \longrightarrow \mathscr{M}_{i}, \quad i=0,1,
$$

which can be regarded as an element

$$
\left(\alpha_{0}, \alpha_{1}\right) \in K^{\prime}:=\operatorname{Ext}_{\left(\mathscr{O}_{0}^{\prime}, x_{1}^{\prime}\right)}^{1}\left(\left(L \zeta_{0}^{\prime *}\left(L_{\mathscr{Y}_{0} / c^{n_{0} \times \mathscr{Z}}}^{\cdot}\right), L \zeta_{1}^{\prime *}\left(L_{\mathscr{O}_{1} / c^{n_{1} \times \mathscr{Z}}}^{\cdot}\right),\left(\mathscr{M}_{0}, \mathscr{M}_{1}\right)\right)\right.
$$

(compare also [Fl] (2.21)(6)). We define ob $\left(\zeta_{0}^{\prime}, \zeta_{1}^{\prime}\right)$ to be the image of $\left(\alpha_{0}, \alpha_{1}\right)$ in $K$ under the canonical map $K^{\prime} \rightarrow K$. The reader may easily verify that this element depends neither on the chosen embeddings of $\mathscr{Y}_{i}$ nor on the liftings $\tilde{\zeta}_{i}$. 
Obviously, if there is an extension $\left(\zeta_{0}, \zeta_{1}\right)$ of $\left(\zeta_{0}^{\prime}, \zeta_{1}^{\prime}\right)$ then ob $\left(\zeta_{0}^{\prime}, \zeta_{1}^{\prime}\right)$ is zero, since we can take $\tilde{\zeta}_{i}:=\zeta_{i}$ and so even $\left(\alpha_{0}, \alpha_{1}\right)$ vanishes.

Conversely suppose that ob $\left(\zeta_{0}^{\prime}, \zeta_{1}^{\prime}\right)$ vanishes. Then there is a pair

$$
\left(\beta_{0}, \beta_{1}\right) \in K^{\prime \prime}:=\operatorname{Ext}_{\left(\mathscr{X}_{0}^{\prime}, \mathscr{X}_{1}^{\prime}\right)}^{0}\left(\left(L \zeta^{\prime *}\left(L_{c^{n_{0} \times \mathscr{L} / \mathscr{Z}}}^{\cdot}\right), L \zeta_{1}^{\prime *}\left(L_{c^{n_{1} \times \mathscr{Z} / \mathscr{Z}}}^{\cdot}\right)\right),\left(\mathscr{M}_{0}, \mathscr{M}_{1}\right)\right)
$$

inducing $\left(\alpha_{0}, \alpha_{1}\right)$ in $K^{\prime}$. Observe that there is a natural exact sequence

$$
K^{\prime \prime} \longrightarrow K^{\prime} \longrightarrow K \text { 。 }
$$

By modifying $\left(\tilde{\zeta}_{0}, \tilde{\zeta}_{1}\right)$ by $\left(\beta_{0}, \beta_{1}\right)$ we may assume that already $\left(\alpha_{0}, \alpha_{1}\right)$ is zero. But then $\tilde{\zeta}_{0}$ resp. $\tilde{\zeta}_{1}$ maps $\mathscr{X}_{0}$ resp. $\mathscr{X}_{1}$ into $\mathscr{Y}_{0}$ resp. $\mathscr{Y}_{1}$ and so we have an extension of $\left(\zeta_{0}^{\prime}, \zeta_{1}^{\prime}\right)$ as required.

We remark that for the proof of the general case one must use resolvents of complex spaces, see $[\mathrm{Fl}]$ or $[\mathrm{B}-\mathbb{K}]$ (I §8).

\section{References}

[B-K] Bingener, J., Kosarew, S., Lokale Modulräume in der analytischen Geometrie, Preprint (1986)

[Dou $\left.{ }_{1}\right]$ Douady, A., Le problème des modules pour les sous-espaces analytiques compacts d'un espace analytique donné, Ann. Inst. Fourier, 16 (1966), 1-95.

[Dou ${ }_{2}$ Douady, A., Le problème des modules locaux pour les espaces $C^{\top}$-analytiques compacts, Ann. Sci. ENS, 7 (1974), 569-602.

[Fl] Flenner, H., Über Deformationen holomorpher Abbildungen, Habilitationsschrift, Osnabruck 1978.

[F-K] Forster, O., Knorr, K., Konstruktion verseller Familien kompakter komplexer Räume, Lecture Notes in Mathematics 705, Berlin-Heidelberg-New York: Springer V. 1979.

[Fr] Frisch, J., Aplatissement en geómetrie analytique, Ann. ENS (4), $\mathbb{1}$ (1968), 305-312.

[Gra] Grauert, H., Der Satz von Kuranishi fur kompakte komplexe Raume, Invent. Math., 25 (1974), 107-142.

[III] Illusie, L., Complexe Cotangent et Déformations I, II, Lecture Notes in Mathematics 239, 283, Berlin-Heidelberg-New York: Springer V. 1971, 1972.

[Pa] Palamodov, V. P., Deformations of complex spaces, Russ. Math. Surveys, 31 (1976), 129-197.

[Pou 1 Pourcin, G., Sous-espaces privilégiés d'un polycylindre, Ann. Inst. Fourier, 25 (1975), 151-193.

[Pou ${ }_{2}$ ] Pourcin, G., Théorèmes de Douady au-dessus de S, Ann. Scuola Norm. Pisa Gl. Sci., 23 (1969), 451-459.

[S-T] Siu, Y. T., Trautmann, G., Deformations of coherent analytic sheaves with compact supports, Memoirs AMS, 238 (1981). 
